\title{
I TRATTATI DEL GELNHAUSEN E DELL' AMEILH IL DIBATTITO SUL CONCILIO COME SOLUZIONE ALLO SCISMA D'OCCIDENTE*
}

\author{
POR \\ GIovann De RigGI \\ Universidad Gregoriana. Roma
}

\begin{abstract}
Resumen
Este artículo examina la carta de Ameilh que remite al obispo de la Iglesia de Worms, Konrad von Gelnhausen, acerca de la doctrina del Concilio para la resolución del Cisma de Occidente (1378-1417).

En realidad son dos tratados, dos eclesiologías diferentes. Gelnhausen establece contra la eclesiología del Concilio una más oligárquica, la eclesiología de Ameilh. El núcleo del discurso es la doctrina de la Iglesia como "congregation fidelium" lo que es identificado por Ameilh como la propia Iglesia romana -Concilio de Papa y Cardenales-según Gelnhausen, el único órgano legal que representa la Universalidad de la Iglesia.
\end{abstract}

\section{Abstract}

This article examines the card. Ameilh's treatises and the parish priest in Worms, Konrad von Gelnhausen's one, about Council's doctrine as a solution to the Schism of the West (1378-1417).

These are two treatises, two different ecclesiologies. Gelnhausen sets against the Council's ecclesiology a rather oligarchical one, the Ameilh's ecclesiology. The heart of the speech is the doctrine of the Church as «congregatio fidelium», which is identified by Ameilh like the Roman Church itself - Pope and Cardinal's Council-, according to Gelnhausen, the only legal organ which represents the Universality of the Church.

\footnotetext{
* Quest'articolo è un estratto della mia tesi per la licenza in Storia della Chiesa conseguita nel giugno 95 presso la Pontificia Università Gregoriana in Roma.
}

Proyeccion internacional de la iglesia hispana Hispania Sacra 51 (1999) 
Con la morte di papa Gregorio XI, avvenuta il 27 marzo del 1378, iniziava una situazione nella vita della chiesa alquanto particolare. Egli fu il papa che aveva riportato, dopo 70 anni, la residenza del papato e della curia romana da Avignone a Roma. Il popolo romano aveva trovato nel papa presente nella città non solo il padre nella fede, ma anche il garante politico ed economico rispetto agli amministratori dei papi avignonesi che non sempre erano riusciti a farsi amare. Questi non volevano certamente farsi sfuggire di mano la soluzione alla cattività avignonese proprio quando la morte di Gregorio XI poteva essere l'occasione propizia ai cardinali francesi per riportare di nuovo la residenza ad Avignone con l'elezione di uno di loro, espressione della maggioranza del collegio cardinalizio. Il popolo romano, già durante i funerali di Gregorio XI e i novendiali che precedettero il conclave, aveva espresso chiaramente il desiderio di avere un Papa romano o almeno italiano. La richiesta fatta ai cardinali era senza mezzi termini: dal conclave doveva uscire un papa romano $o$ almanco italiano. In questa situazione si svolge il famoso conclave del 7-9 aprile del 1378 , la cui validità sarà da lí a poco messa in discussione da una parte degli stessi cardinali che votarono papa l'arcivescovo di Bari, Bartolomeo Prignano. La conformazione del collegio cardinalizio, la pressione del popolo romano durante l'elezione di Urbano VI, il carattere di quest'ultimo, l'interesse personale dei cardinali, sostenuto da una visione ecclesiologica che li vede come pars capitis o pars corporis papae, divennero motivi sufficienti per una seconda elezione pontificia, avvenuta il 20 settembre successivo, e quindi per lo scisma nella Chiesa del tardo medioevo.

Una volta tentate tutte le strade senza alcun esito per trovare una soluzione allo scisma, quella della via concilii sembrò essere l'unica che desse una garanzia maggiore: l'intera Chiesa, ora, potrà esprimersi sui due pretendenti alla sede di Pietro per ritornare a quella conciliazione tanto attesa. La proposta dei cardinali italiani divenne una vera e propria esplosione di una vivace discussione da parte di teologi e di giuristi per poter giustificare la convocazione del concilio come organo della chiesa, in un momento in cui essa non ha più un capo universalmente riconosciuto. Ed è proprio a questo punto, che l'ecclesiologia si fa ancora più interessante attraverso un continuo dibattito che vede confrontarsi pensieri teologici differenti. La proposta di un'eventuale convocazione conciliare suscitò, soprattutto nei cardinali della prima ora di obbedienza clementista, un'opposizione di carattere teologico e giuridico, a cui solo un'equilibrata risposta poteva tener fronte e giustificare il concilio come unica via possibile per uscire dal tunnel della divisione.

In questo acceso dibattito riaffiorano antiche concezioni ecclesiologiche, in particolare circa la posizione del papa e il suo rapporto con l'intera chiesa. L'ecclesiologia dei secoli passati trovò in questo breve arco di tempo una fe-

Proyección internacional de la iglesia hispana Hispania Sacra 51 (1999) 
conda sollecitazione per risolvere questioni rimaste insolute o che non si immaginava dovessero mai interessare. Si vuole comprendere pienamente cosa significhi il primato del papa all'interno della chiesa, corpo mistico, formato da caput e membra. Così accanto all'ecclesiologia del primato petrino andò sviluppandosi una problematica suscitata dalla situazione storica che vide una piena partecipazione delle membra alla vita dello stesso corpo mistico di Cristo. Il confronto, allora, interessò due pensieri impegnati su un unico tema di interesse generale e tentò un'esegesi di decreti e decretali che col passar del tempo erano andate elaborandosi ponendo elementi teologici nella legiferazione del Corpus juris canonici. Lo sviluppo dell'ecclesiologia e pari passo anche quello della tematica conciliare trovò successivamente durante lo scisma occidentale un motivo ulteriore per un nuovo contributo alla ricerca giuridicoteologica intorno a un particolare problema come quello che in quegli anni andò creandosi nella chiesa di Roma e che interessava la chiesa universale. I 39 anni del periodo dello scisma segnaronó non solo il cammino della chiesa ma fecero da stimolo all'ecclesiologia che precedentemente aveva già puntualizzato alcune questioni particolari che ritorneranno in tutti gli autori che in quel determinato momento cercarono di trovare alcune soluzioni di carattere guiridico inquadrate in una visione più generale sulla natura stessa della chiesa. Si potrebbe dire che proprio interessi di carattere prevalentemente giuridico faranno da sfondo a un tipo di ecclesiologia.

In questo dibattito si pongono i due trattati dell'Amielh e del Gelnhausen. Espressione di una diversa teologia ecclesiologica, modelli di due teologie a confronto, essi sono esempi chiari dei problemi che l'ecclesiologia precedente non aveva ben chiarito. Dal decreto di Graziano in poi una fitta produzione di opere a carattere teologico e giuridico tentarono di dare un'interpretazione del primato accanto all'altra visione ecclesiologica che poneva il collegio cardinalizio come compartecipe all'esercizio della plenitudo potestatis del papa. Se i trattati dell'Ameilh e del Gelhnausen possono essere letti come modelli di questo dibattito, è pur vero che essi sono il risultato di una ecclesiologia precedente, dove l'una poneva l'accento sul primato petrino nella chiesa in modo da poter garantire alcuni privilegi, l'altra invece cercava una via di equilibrio per comprendere la vera costituzione di essa, senza tuttavia negare il primato del papa. Rappresentano rispettivamente un'idea ecclesiologica che già antecedentemente, soprattutto nel corso della prima metà del secolo XIV, andò formulandosi attraverso un dibattito stimolato da vicende storiche che generò un confronto di tipo dottrinale sull'antica questione del rapporto tra sacerdotium et imperium. Il risultato delle ricerche teologiche e giuridiche da questo fervido e accanito confronto servì a porre le basi al futuro sviluppo della tematica ecclesiologica. Sia 1'Ameilh che il Gelnhausen si pongono in questa linea di pensiero che generò diverse categorie concettuali, sorte per dare risposte 
concrete a questioni di carattere prettamente giuridico-teologico che toccavano argomenti che interessavano problemi «intra ecclesiam» o «extra ecclesiam» cioè il rapporto tra la Chiesa, in particolare il papa, e l'imperatore o il principe nel proprio regno.

I trattati dell'Ameilh e del Gelnhausen, dunque, prendono le mosse dai precedenti trattati ecclesiologici. Si potrebbe dire che questi due autori possono essere presi quasi ad esempio per confrontare cosa abbia prodotto il risultato di un Iungo procedimento di riflessione teologica che partendo dal medioevo e soprattutto dalla formulazione del Decretum trovò proprio nel secolo XIV un periodo denso di sollecitazioni per le vicende storiche. L'ecclesiologia di quel periodo, infatti, fu intimanente connessa al «fatto» storico. In tutto il secolo XIV Ia riflessione sull'ecclesiologia fu motivata da un dialettico confronto sui rapporti tra sacerdotium et imperium. All'antica questione si presentò una nuova: per imperium non si intese solo lo spazio d'influenza dell'imperatore ma anche quello del singolo principe nel suo regno, in quel processo di formazione degli Stati nazionali proprio di quest'epoca.

Ma accanto al problema dell'ecclesiologia «extra ecclesiam», si presentò quello «intra ecclesiam». Il secolo XIV segnò anche il periodo in cui la visione ecclesiologica, che vedeva i cardinali con un ruolo importante e di primaria importanza, assunse caratteri maggiormente forti all'interno sia della trattazione propriamente teologica o giuridica, ma soprattutto nella convinzione del collegio cardinalizio stesso che ebbe una parte predominante nello scisma della chiesa. Per cui, come vedremo particolarmente in seguito, la visione della chiesa e soprattutto l'esercizio dell'autorità nel suo interno non aveva ancora una chiara strutturazione, dovuta soprattutto a idee e sviluppi che hanno dato adito a comprensioni errate: non si trattava tanto di toccare la natura stessa della chiesa, quanto quello dell'esercizio dell' autorità all'intemo di essa e all'esterno.

In quest'orizzonte si pone il discorso sul concilio e sul suo posto all'interno della Chiesa. Quasi sempre il concilio fu visto accanto al potere del papa o addirittura superiore a lui (conciliarismo). Eppure la stessa visione conciliare aveva premesse propriamente ecclesiologico-giuridiche che affondano radici nel medioevo, spuntano nel dibattito del XIV secolo e si ritrovano puntualmente nel periodo dello scisma. Gelnhausen si pone in questa scia con alcune fondamentali differenze, mentre l'Ameilh presenta un'altra visione che tende a salvaguardare i diritti dei cardinali contro ogni tentativo di ridimensionamento che un concilio poteva attuare. 


\section{Il trattato di PETRUS AMELII.}

Lo scritto del cardinale Petrus Amelii' ${ }^{1}$, Ameilh, Amiel, si pone in questa raccolta di trattati ecclesiologici di tipo teologico-giuridico in modo abbastanza fedele con la visione che dal Flandrin in poi è stata data come vero problema per attaccare l'idea del concilio. Tuttavia, il suo scritto, pur inserendosi in questa raccolta di testi anticonciliaristi di carattere giuridico-teologico, presenta un aspetto particolarmente pratico; vuole essere, infatti, una vera e propria risposta a coloro che invocano il concilio, teologi o politici che siano, adducendone motivi non del tutto sostenibili. Il suo Contra petentes concilium generale $^{2}$ è, dunque, espressione del pensiero giuridico-teologico in cui il primato del papa e quello dei cardinali è messo ben in evidenza.

Tra l'agosto del 1379 e il maggio del 1380 nella residenza avignonese, dove ha seguito Clemente VII e trascorso gli ultimi anni della sua vita, l'Ameilh si dedicherà a questo trattato contro il progetto di un concilio generale, come egli stesso espressamente afferma all'inizio dello scritto: «Quoniam a pluribus asseritur, quod per universale concilium cognosci debet et decidi de iure vel non iure Bartholomei se pro papa Romano gerentis», in quanto il suo lavoro dovrà servire a confutare esplicitamente questo progetto che ormai si faceva sempre più strada. Il re di Francia, Carlo V, alcuni cardinali, l'Università di Parigi erano dell'opinione di una convocazione generale per decidere la verità dell'uno o dell'altro papa. Ma forse il motivo più profondo dell'origine di quest'opera è da ritrovarsi nell' Epistola brevis che il preposto di Worms, Konrad von Gelnhausen, aveva inviato a Carlo V di Francia su sua richiesta riguardo al concilio come unica possibilità per poter uscire dal problema dello scisma. Questa lettera è del 31 agosto del 1379. In questo stesso periodo un altro cardinale, Flandrin, aveva scritto un trattato in duplice parte, in cui riportava gli episodi dei gioni dell'elezione del Prignano e le motivazioni per ritenere invalida quell'elezione. Certamente questo scritto doveva essere conosciuto dal Gelnhausen, mentre non sembra conosca quello dell'Ameilh, motivo più valido per ritenere che quest'ultimo ha messo mano alla sua opera solo

I Benedettino, prima di essere creato cardinale da Clemente VII nel dicembre del 1378, fu Abate di S. Benigne de Dijon* Fu nominato poi vescovo di Vienne il 27 aprile 1362 e l'anno successivo fu trasferito alla sede arcivescovile di Napoli dove fu particolarmente impegnato in ambascerie pontificie per la pace nel regno napoletano e per la soluzione della questione dei confini del territorio beneventano. Divenne infine vescovo di Embrun, per cui una volta cardinale sarà sempre chiamato il cardinale Embrunensis. Morirà il 10 agosto del 1389, lo stesso anno della morte di Urbano VI. Cfr G. MOLLAT, Amiel Pierre, in DHGE, II, 1252-1253.

2 Il trattato è in FR. BLIEMETZRIEDER, Literarische Polemik zu Beginn des grossen abendländischen Schismas, Wien-Leipzig 1910 (rep.1967) 91-111, con un'introduzione alle pagine 61*-70*. D'ora in poi Tractatus e pagina. 
dopo l'agosto del 1379, conoscendo bene l'Epistola del preposto di Worms. Il trattato dell'Ameilh farà lavorare di nuovo il Gelnhausen per la sua Epistola concordiae, trattato di risposta molto più completo e ricco del primo, di cui parleremo appresso. Così dall'agosto del 1379 al maggio del 1380 si deve porre il tempo di composizione del Contra petentes.

Il metodo usato dall'Ameilh è molto interessante. Essendo un'opera di confutazione non poteva essere scritta con un metodo discorsivo. Il suo trattato è costruito col metodo scolastico: enunciazione del tema, obiezioni, conclusione dell'autore. All'Embrunensis interessa principalmente chiarire i punti su cui $\mathrm{i}$ plures fanno leva per ritenere possibile la convocazione del concilio, per cui non sembra esserci altro metodo più efficace di questo, per ottenere quanto si desidera. Così il trattato si struttura in otto conclusioni che si muovono su un piano teorico e pratico ${ }^{3}$. Presenta spiegazioni alquanto dotte e interessanti, che abbracciano l'aspetto teologico, giuridico e infine anche quello storico ${ }^{4}$. Inoltre, la progressione delle conclusioni presenta una logica interna. Non vengono messe una accanto all'altra disordinatamente, ma sono poste in modo tale da ottenere una progressiva linea di pensiero ben preciso, tanto da risultare un lavoro composto da una composizione in una triplice struttura: tema iniziale, corpo, conclusione. Il punto principale su cui l'intervento doveva essere più forte si poneva con più vigore: il concilio non può essere convocato senza l'autorità del papa, per di più contro il papa. A questo tema principale, fa seguito il corpo del discorso che approfondisce ulteriormente quanto detto prima, fino ad arrivare alla conclusione che ha un aspetto pratico e teorico. Teorico: $\mathrm{i}$ cardinali sono la Chiesa Romana che è la Chiesa universale, conseguenza logica (aspetto pratico): non c'è bisogno del concilio come espressione della chiesa universale, la loro decisione vincola tutti i fedeli. Per cui, il motivo principale dell'intera opera è duplice: da una parte affermare che $\mathrm{i}$ signori cardinali hanno il potere di dichiarare chi è il vero papa, e dall'altra sostenere che Clemente VII è il papa legittimo. Così una convocazione conciliare non solo sarebbe impossibile per ordine giuridico-teologico, ma anche inutile.

Contro l'idea di convocare un concilio da parte dei cardinali, di imperatori, di re di tutta l'intera chiesa, in quanto il potere dato da Cristo a Pietro come capo è passato a tutto il corpo, l'Ameilh impugna la penna ${ }^{5}$. Arriva subito alla prima conclusione: i prelati senza l'autorità della Sede apostolica non possono celebrare nessun concilio. Se qualsiasi concilio venisse celebrato senza il Ro-

3 Cfr Fr. BLIEMETZRIEDER,op. cit., 65*.

4 Sembra che lo stesso Gelnhausen abbia apprezzato qūesto modo di scrivere dell'Ameilh, quando nella sua Epistola concordiae parla di tractatus subtiles che escogitano ogni difficolta giuridiche e teologiche per la convocazione del concilio.

\$ Tractatus, 92.

Proyección internacional de la iglesia hispana Hispania \$acra 51 (1999) 
mano Pontefice, è da considerarsi non un vero concilio ma un conciliabulum che non ha nessun effetto in quanto nullo, irritum, e neppure la convocazione di tutti i vescovi si può ritenere espressione della plenitudo potestatis, perchè è prerogativa della sola Chiesa Romana ${ }^{6}$. Non c'è dunque nessun motivo valido perchè ci si arroghi il diritto di convocare il concilio, in quanto, e qui la seconda conclusione: Synodus universalis...papam iudicare non posset ${ }^{7}$.

Il punto centrale della discussione tra teologi e giuristi si fa ormai presente. Una volta assodato che senza il papa non si può convocare nessun concilio, ora l'Ameilh tocca l'altro grande punto del programma di coloro che lo vogliono: il giudizio sul papa. Ciò non è possibile perchè Nemo iudicabit primam sedem. Neque enim ab augusto, neque ab omni clero, neque a populo, nè tanto meno un concilio che non può stabilire nulla perchè privo dell'autorità papale. Il concilio col papa non statueret, sed suaderet. Dunque, nessun inferiore può mai giudicare il papa che gode della pienezza di potestà ${ }^{9}$. Nè tanto meno il papa può sottomettersi al giudizio del concilio ${ }^{10}$. Da una lunga lista di episodi storici, in cui l'Ameilh riporta le varie condanne fatte da papi ad altri pretendenti e intrusi nella sede apostolica ${ }^{1 !}$, trae questa conclusione:

6 Interessante questo punto del trattato. In molti teologi era valsa l'idea derivante dall'interpretazione di Mt 16, 18, in cui il primato dato da Gesù Cristo si legge come dato all'intera chiesa per cui l'insieme dei pastori delle varie comunità in un concilio garantirebbe la plenitudo potestatis su tutta la chiesa. L'Ameilh afferma che la potestas del vescovo è sulla chiesa di cui egli è pastore e solo su essa. Tutti i vescovi insieme non danno la pienezza della potestà perchè ognuno ha potestà super singulos subditos,cfr Tractatus, 93.

7 lbidem, 93 .

8 Sentenza del decreto di Graziano: Causa IX, q.3, cap. XIII.

9 L'Ameilh,a prova di quanto afferma, riporta il modo di definizione dei vari documenti dei concili: Nos itaque: cum fratibus nostris et sancto concilio nostro deliberacione prehabita, de consilio concilii. Invece, le espressioni come: Placuit sancto concilio sono il segno dell'assenza del papa dal concilio. Ma quando iI papa interfuit al concilio, è sempre la sua autorità prima della stessa assemblea conciliare ad essere tenuta in considerazione.

10 Ibidem, 94.

11 Si tratta: 1. dello scisma laurenziano con T'elezione nello stesso giomo del diacono Simmaco e dell'arciprete Lorenzo fino a che Teodorico il Grande non si decise a favore di Simmaco che fu papa dal 498 al 514. In seguito i fautori di Lorenzo convinsero l'imperatore di gravi accuse contro papa Simmaco. Si convocò un sinodo di vescovi a Roma, ma nella $4^{3}$ sessione dichiaró di non poter giudicare la sede apostolica. 2. Marcellino (296-304) che secondo il Liber pontificalis avrebbe offerto incenso durante la persecuzione di Diocleziano e in seguito mori martire. Fu accusato fortemente di apostasia dal vescovo donatista Petiliano, ma difeso da Sant'Agostino. 3. Damaso (366-384) che condannò il modo di fare di papa Liberio con la sottoscrzione della formula di fede emanata da Costanzo su istigazione del partito ariano con la scomunica di Atanasio e Felice creato papa dallo stesso imperatore. 4. Stefano III (768-72), papa dopo aver deposto l'intruso Costantino elefto papa da suo fratello Toto di Nepi. 5. Stefano VI (896-97), per ordine del duca di Spoleto, fece esumare il corpo del papa Formoso dichiarando illegittimo il suo pontificato e tutti i suoi atti. In seguito i papi Teodoro $\Pi$ e Giovanni IX annullarono gli atti del Synodus ad cadaver. 6. Giovanni XII (955-964) scomunicò 


\begin{abstract}
«Ex quibus apparet, quod in casu nostro inutiliter vocaretur concilium ad decernendum de non jure $B$ [artholomei], quia si ipse papa non est, concilium eius auctoritate celebrari non potest, et sine concilio dominus noster Clemens ipsum condempnare potest, sicut et fecit; si vero esset, concilium de papatu suo iudicare non posset»12
\end{abstract}

Solo il papa, dunque, può giudicare. Quello vero è Clemente VII, per cui solo lui può dichiarare illegittimo il Prignano, come ha fatto. Urbano VI, se considerato vero papa, non potrebbe convocare un concilio in cui si metterà in discussione la sua elezione, e quindi il suo pontificato ${ }^{13}$, sarebbe un controsenso. Neppure il problema del papa dubbio potrebbe giustificare il concilio, perchè in ogni caso ci sarebbe una sottomissione del papa all'assemblea concilia$\mathrm{re}^{14}$, cosa impossibile a farsi. Qualsiasi concilio che venga convocato per questo motivo non può essere che un conciliabolo, per cui non ha nessun valore giuridico per la chiesa. Nel caso particolare questo principio vale ancora di più, perchè essendo Bartolomeo Prignano arcivescovo di Bari e ufficiale della chiesa romana è soggetto direttamente al papa, dunque a Clemente VII che era cardinale di S. Romana Chiesa.

Da questo punto in poi, l'Ameilh sposta la questione dal potere del concilio a quello del papa. Ormai, ogni giudizio è posto nelle mani del papa che gode della plenitudo potestatis. La terza conclusione ripete praticamente lo stesso concetto, in quanto se il concilio può giudicare un papa eretico notoriamente, automaticamente non si deve a lui più obbedienza. Ora, la stessa cosa deve avvenire per il Prignano. Se lo si giudica in concilio non può essere più ritenuto papa, perchè è stato sottoposto al giudizio conciliare. Anzi, lui stesso potrebbe ritrattare la sentenza conciliare qualora risultasse a lui sfavorevole, quia papa solus potest de sententia concilionum iudicare ${ }^{15}$. Inoltre, tutto questo lavoro sarebbe inutile: Bartolomeo Prignano non è papa. Con la quarta conclusione l'Ameilh nota che un eventuale concilio difettoso nella sua natura giuridica non potrebbe in alcun modo legare giuridicamente i sudditi della chiesa, così la quinta conclusione ricorda che l'unico modo di elezione del papa è l'accordo delle due parti dei cardinali. Termina, così, la prima parte del tratta-

\footnotetext{
l'antipapa Leone VU nel sinodo del 964 a Roma. Per questo l'Ameilh porta a sostegno della sua tesi episodi storici che attestano giudizi di Papi su altri pretendenti o su operato di papi legittimi. Ma la spiegazione non regge affatto. Qui ci troviamo davanti ad un fatto unico nella storia della Chiesa.

12 Ibidem, 95.

13 A rigor di logica il discorso dell'Ameilh regge benissimo. D'altra parte già da tempo si ci poneva il problema di chi potesse convocare il concilio. Farlo convocare da qualcuno dei due papi significava implicitamente riconoscerlo come tale. Ma un concilio convocato da uno dei due non può in seguito discutere della sua legittimità e del șuo pontificato. Il problema era ben chiaro.

14 lbidem, 97.

15 Ibidem, 99.
}

Proyección internacional de la iglesia hispana Hispania Sacta 51 (1999) 
to, in quanto ciò che segue sarà solo una particolare considerazione sulla situazione politica del tempo che difficilmente potrebbe essere vero aiuto per una serena conduzione del concilio, fino ad arrivare alla conclusione in cui l'Ameilh tratta della posizione dei cardinali nella chiesa e quindi della loro unica voce in capitolo per risolvere la spinosa situazione della chiesa.

In questa prima parte, l'Embrunensis ha voluto subito chiarire la sua posizione circa il papa e la convocazione del concilio sia a livello dottrinale che giuridico. Moltissime situazioni storiche, come abbiamo notato, vengono addotte per chiarire ulteriormente la situazione attuale. In ogni caso, il pensiero fondamentale che pervade l'intero scritto e su cui viene a costruirsi è che Clemente VII è il vero papa, che non può essere giudicato dal concilio. Urbano VI non è altro che un usurpatore e dev'essere giudicato direttamente dal Papa in quanto occupante della sede di Roma. Il caso del duplice papa non viene tenuta in debita considerazione. Per lui non esiste che un unico papa che è Clemente VII che ha il potere di giudicare l'usturpatore e l'intruso sulla cattedra di Pietro. Così si spiegano i numerosi episodi storici portati come esempio e giustificazione della sua idea. In tutti questi esempi si trova un unico motivo: il vero papa condanna e depone quello falso ${ }^{16}$.

Per la sesta conclusione ${ }^{17}$ sono da notare due elementi essenziali: da una parte l'Ameilh esamina la situazione politica e il problema del luogo della celebrazione del concilio, dall'altra invece, richiamando la lotta tra Alessandro III e l'imperatore Federico I Barbarossa, arriva alla settima conclusione per spiegare che la convocazione del concilio militat contro la libertà della chiesa. Cosi l'Ameilh controbatte i petentes concilii proprio su due punti pratici: luogo del concilio e autorità della convocazione ${ }^{18}$. Quest'ultimo punto era di interesse generale. Non si poteva chiedere all'uno o all'altro papa di convocare il concilio, perchè sarebbe stato un implicito riconoscimento dela sua legittimità. L'unica via era quella di chiedere la convocazione al potere politico. L'imperatore o qualsiasi altro re, in quanto difensore della fede e della chiesa

16 Un fatto analogo allo scisma d'Occidente potrebbe essere quello del 1130-1138 con la duplice elezione di Innocenzo II e di Anacleto II, quando in Roma nello stesso giorno si procederte ad una duplice elezione. Solo l'intervento autorevole di S. Bernardo fece confluire l'intera obbedienza della chiesa per Innocenzo II. In ogni caso, non si può considerare Anacleto II come antipapa. I metodi usati dalla famiglia dei Pierleoni col cardinale Gregorio restano alquanto inspiegabili.

17 Tractatus, 101.

18 Sembra che nessuna questione sia sfuggita all'Embrunensis. La sesta conclusione non ha solo un carattere di tipo pratico. La risposta a coloro che pensavano di trovare nell'imperatore l'autorità giusta per la convocazione del concilio è scartata. Il racconto dell'episodio del 1160, quando Federico I convoca a Pavia un sinodo per risolvere la contesa ecclesiastica tra Alessandro III e il «suo» papa Vittore IV, permette di evidenziare che qualsiasi ingerenza nella chiesa del potere politico sarebbe motivo di mancanza di liberta. 
poteva intervenire per il bene di essa. Invece, l'Ameilh ribadisce che il concilio puó essere convocato solo dal papa come è avvenuto per altri casi di scisma non però per discutere l'utrum sit verus papa, ma per altri motivi ${ }^{19}$; ecco l'ottava conclusione. Come sempre a sostegno di questa tesi adduce ancora altri casi storici ${ }^{20}$ con una personale lettura interpretativa. Il sinodo viene visto come una possibilità in cui il vero papa può dichiarare scomunicato e intruso colui che la storia definisce antipapa.

Resta ancora un altro argomento da trattare quello sui cardinali. Chi sono e che compito possono avere in questa determinata situazione? Prima di tutto, l'Ameilh pone una precisa questione: $\mathrm{i}$ cardinali hanno una propria giurisdizione? Sembra di si, per diverse ragioni:

- hanno nella chiesa una propria dignità con particolari prerogative;

- in alcune questioni hanno particolare connessione col papa e senza di lui facoltà proprie;

- hanno una propria giurisdizione;

- hanno piena potestà per l'elezione del pontefice e per la sua approvazione;

- hanno diritto d'essere consultati per tutte le questioni ecclesiastiche;

- in caso di scisma sono loro a dichiarare la verità.

La conseguenza è facilmente intuibile. Spetta ai cardinali, ora, fare la dichiarazione su chi dei due sia il vero papa. Non c'è alcun bisogno di convocare un concilio perchè essi hanno quest'autorità nella chiesa. Autorità che non è stata diminuita dalla Ne Romani di Clemente V. Essa ha solamente proibito di esercitare il potere proprio del papa durante la sede vacante ma non quello proprio dei cardinali e inoltre non aggiunge nessun'altra prerogativa, ma richiama solo 1'antico diritto proprio dei cardinali ${ }^{21}$. Infine, anche 1'ultimissimo aspetto della universalità della chiesa addotta dai «conciliaristi» come uno dei punti più importanti per giustificare la convocazione del concilio è risolto dall'Ameilh. La chiesa universale viene fatta coincidere con la chiesa di Roma.

19 «...quod tempore scismatis ille qui est verus summus pontifex potest convocare concilia particularia prelatorum aliquorum regnorum, nunc hic, nunc alibi, non quidem ad discuciendum utrum ipse sit verus papa, sed super aliis occurrentibus negociis de quibus expediens sibi videtur, et quandoque ut magis declaretur et stabiliatur ius suum», 105.

20 Niccoló II eletto a Siena nel 1058, mentre a Roma dalla nobiltà romana fu eletto Benedetto X, convocò un sinodo a Sutri dove condannò l'antipapa. Alessandro II (1061-1073), papa, mentre a Roma fu eletto il vescovo di Parma Cadalo col nome di Onorio II, convocò un sinodo in Lombardia dove dovette chiarire della sua elezione. Infine riporta il caso dello scisma del 1130-1138, di cui abbiamo parlato sopra,

21 Tractatus, 109.

Proyección internacional de la iglesia hispana

Hispania Sacra 51 (1999) 
Essendo i cardinali nella chiesa di Roma, anzi essendo loro stessi la chiesa di Roma $^{22}$, il collegio cardinalizio è la chiesa universale, per cui quell'universalità che il concilio potrebbe esprimere è garantita e mantenuta dal collegio dei cardinali che hanno una propria autorità. Dunque, il vero papa è Clemente VII e in conseguenza la convocazione di un concilio sarebbe impossibile ma soprattutto inutile.

Da una lettura attenta di questo trattato risulta un intento preciso dell'autore in una duplice direzione. All'Ameilh interessa da una parte confutare l'idea conciliare, dall'altra affermare che Clemente VII pud essere serenamente riconosciuto come papa. Parte da una adesione che in un certo senso aprioristicamente potrebbe inficiare la validità della teoria esposta nel suo scritto. La sua non sembra essere una serena esposizione della dottrina teologico-giuridica del primato del papa e dell'autorità del concilio, quanto invece una strenua difesa della posizione di Clemente VII strutturata con elementi di carattere teologicogiuridico per chiarire l'impossibilità del concilio e l'invalidità di esso qualora venisse celebrato. La sua posizione non gli fa comprendere appieno la situazione particolare della chiesa e il desiderio di risolvere lo scandalo dello scisma. Così l'interesse per la difesa dell'impossibilità di giudizio del papa non lo fa entrare nel cuore stesso del problema: qui non si tratta di voler deporre un papa e quindi di giudicarlo, quanto invece del dubbio sui due pretendenti alla sede romana. Aver accettato Clemente VII come unico e vero papa significa considerare illecito e invalido ogni concilio che voglia giudicarlo e deporlo, proprio come la tradizione della chiesa e il Decretum di Graziano aveva ribadito. Lo stesso utilizzo della storia sembra essere alquanto insufficiente. Molte volte abbiamo ribadito che lo scisma di questo periodo non può trovare nessun riscontro in altri scismi consumati nel lungo corso della storia della chiesa. Quasi tutti gli esempi addotti dall'Ameilh, tranne quello dello scisma del 1130-1138, sono dovuti a veri e propri antipapi che o dalla nobiltà romana 0 dal potere imperiale venivano messi a regnare sulla chiesa di Roma. Ora invece, il problema è un altro: quale dei due può essere ritenuto il papa legittimo, visto che entrambi possono addurre motivi validi per giustificare la propria legittimità? Nessun riscontro poteva trovarsi nella storia della Chiesa.

Un altro aspetto ancora potrebbe essere facilmente confutabile, oggetto della sesta conclusione: nessun potere politico potrebbe entrare nelle questioni ecclesiastiche, pena la mancanza di libertà della chiesa. Se in linea di principio ciò è verissimo, nella situazione dello scisma di questo periodo è alquanto falso. Studi approfonditi sullo scisma hanno messo ben in evidenza come lo schieramento politico per l'uno o l'altro papa sia stato il garante del perdurare

22 «Plus dico quod papa et domini cardinales ita sunt in Romana ecclesia, quod exiam ipsi sunt eadem Romana ecclesia, que claves accepit et potestatem ligandi atque solvendi», 110 . 
dello scisma. Le due obbedienze erano garantite dalla scelta politica dei re e dell'imperatore, anche se questi in un primo momento sembravano assumere un atteggiamento di estrema prudenza. In questo senso è da vedersi la decisione del sinodo del clero di Parigi del 1398 di ritirare l'obbedienza a Benedetto XIII. Esso aveva veramente colto il cuore del problema. L'appoggio politico serviva all'uno e all'altro. Porre il problema della mancanza di libertà, allora, sembra essere poco plausibile.

Lo scritto dell' Ameilh, in ogni caso, si presenta con una solidità metodologica e con una evoluzione concettuale ben precisa. Lo stesso metodo scolastico permette di non perdersi nella trattazione della materia, ma di avere una unica linea di argomenti validi per confutare il progetto generale del concilio. Dal papa ai cardinali, gli unici che possono garantire nel dubbio della decisione, si snoda I'intero scritto dell'Embrunensis. Non mancano tuttavia alcuni accenni di particolare ironia nei confronti del Prignano. Il suo trattato come quelli degli altri difensori di Clemente VII è un tentativo di accurata esposizione giuridica più che teologica sulla natura della chiesa, in cui gli elementi principali della trattazione restano l'impossibilità di giudizio sul papa e la posizione privilegiata dei cardinali all'interno di questa unica partecipazione alla plenitudo potestatis che è della chiesa romana e dunque del papa e del collegio cardinalizio con lui. Del problema tipicamente ecclesiologico, tratteremo più avanti.

In conclusione, rispetto all'Epistola brevis del Gelnhausen, to scritto dell'Ameilh presenta tutte le caratteristiche della speculazione giuridica del tempo. Pur essendo un trattato relativamente breve, tuttavia presenta il vantaggio di un'esauriente visione giuridica ed ecclesiologica in una sintesi molto chiara. L'unico punto negativo è il non aver compreso o non aver voluto comprendere la particolare situazione: il dubbio sui due come possibilità di una convocazione conciliare.

Questo trattato non poteva restare senza risposta. Il Gelnhausen, nel maggio del 1380 «risponde» con una nuova Epistola.

\section{L'EPISTOLA CONCORDIAE DI KONRAD VON GELNHAUSEN.}

Il preposto di Worms, Gelnhausen ${ }^{23}$, è uno dei più importanti rappresentanti della teoria conciliare del periodo dello scisma d'Occidente, insieme a Heinrich di Langenstein e al Gerson. Pur tuttavia, non può essere annoverato tra coloro che protendono esplicitamente verso il conciliarismo, che è tutt'altra cosa rispetto all'idea conciliare che per questo determinato problema si va

23 Scarse notizie biografiche si hanno in G. MOLLAT, Conrad de Gelnhausen, in DHGE 484-485.

Proyección internacional de la iglesia hispana Hispania Sacra 51 (1999) 
sviluppando. Il trattato del Gelnhausen ${ }^{24}$ può essere esplicitamente denominato come una risposta chiara, sollecitata dallo scritto dell'Ameilh. Mentre con la sua Epistola brevis del 31 agosto 1379 inviata a Carlo V di Francia Gelnhausen risponde alla stessa richiesta del re tramite una lettera in cui si prospetta la teoria del concilio per risolvere il problema dello scisma, questo trattato non nasce da una richiesta esplicita dal parte del re, ma è mossa dall'esigenza di dare una vera e propria risposta a tutti coloro che non accettavano l'idea del concilio, adducendo problemi più di carattere giuridico che dottrinali. Il suo trattato ha di mira il Flandrin, il de Berreria e in particolare lo stesso Ameilh, il cui scritto voleva essere una vera risposta all'Epistola brevis.

Il merito del Gelnhausen è stato quello di aver strutturato il suo testo in base ad argomenti giuridici e teologici. Presenta, inoltre, un aspetto particolarmente pratico nella sua richiesta ${ }^{25}$. ma la sua idea è sostenuta non solo da una solida preparazione storica ma soprattutto teologica. Il Langenstein ${ }^{26}$, invece, è più propriamente teologo, per cui le sue sońo speculazioni alquanto libere con una fondata sintesi teologica. D'altra parte il Gelnhausen non poteva muoversi solo sul campo teologico, in quanto il suo scritto doveva essere una vera e propria confutazione delle tesi esposte dalla parte contraria $\mathrm{i}$ cui punti erano essenzialmente due: storia e diritto. Un punto a vantaggio del Gelnahusen è di esser andato in profondità rispetto ai suoi oppositori. Mentre questi sollevavano problemi quasi esclusivamente di carattere giuridico, il Gelnahusen propose un'idea ecclesiologica alquanto singolare che non solo servi al momento dello scisma, ma anche ad una più profonda comprensione della chiesa.

Il metodo usato dal Gelnhausen, quindi, è differente da quello utilizzato dall'Ameilh. Mentre questo procedeva in modo scolastico, il Gelhnausen procede argomentando in modo discorsivo senza abbandonare del tutto il tipico procedimento dell'argomentazione scolastica. Dal principio espone la sua tesi: il concilio può e dev'essere convocato, se si vuole risolvere il problema del miserabile ac contagiosissimum scisma. Così, l'Epistola concordiae a Carlo $\mathrm{V}$ di Francia del maggio 1380 è composta da quattro capitoli, in cui Gelnhausen ripropone in modo più fondato l'idea che già aveva espresso nella sua precedente Epistola bervis. I quattro capitoli sono in continuo procedimento che va

24 Anche per quest'opera mi servo dell'edizione critica in FR. BLIEMETZRIEDER, op. cit.,111-140, con un'introduzione interessante alle pp. 71*-91*. D'ora in poi Epistola con pagina di riferimento.

25 «Que quidem benedicta pax et unitas salubriter reformari et scisma miserabile ac contagissimum de medio auferri posse non videtur aliqua via mundi,circumspecto tamen miraculo divino, nisi per viam concilii generalis, prout alias regie magnificencie dixi oraculo vive vocis, et postdum de serenitas vestre mandato in quadam brevi epistola regali maiestati transmissa non ut decuit sed prout minus male exaravi», Epistola, 113.

26 If Langenstein ha scritto l'Epistola concilit pacis con cui si allinea alla schiera di coloro che progettavano il concilio come soluzione allo \$cisma d'Occidente. 
dall'affermazione della sua tesi fino alla confutazione delle opinioni contrarie, contrarium sententiae di quidam magni et famosi e di domini magni et periti valde. Inevitabilmente il riferimento è a coloro che hanno usato tutta la scienza giuridica per ostacolare il progetto conciliare. Il trattato può essere diviso in due fondamentali parti: I e III capitolo e II e IV capitolo. Nel primo e terzo capitolo, Gelnhausen espone la sua idea circa il concilio e la Chiesa, mentre il secondo e il quarto sono rispettivamente enunciazioni e risposte mosse dalla parte contraria. Inoltre, la duplice introduzione della lettera ${ }^{27}$ ha come oggetto principale la richiesta del concilio, unica soluzione al dramma della madre chiesa. Scrivendo a Carlo V, il preposto di Worms afferma:

«Quis ego sum, aut que vita mea, vel que cognacio patris mei, ut scribam regibus, aut eciam repugnem legibus!»28,

il che fa presupporre una richiesta da parte del re. Certamente questa avvenne prima dell' agosto del 1379 e la risposta fu l'Epistola brevis, per la presente non si può affermare del tutto se ci sia stata un'altra richiesta del re. Il motivo della confutazione del trattato dell'Ameilh è invece molto più probabile.

Interessante in questa introduzione notare il concetto di chiesa esposto dal Gelnhausen. La chiesa si presenta come una Madre spirituale, deturpata dallo scandaloso scisma e divisa in se stessa. Questo scisma è gioia per i Saraceni, tripudio per i Giudei e via all'Anticristo. La vita cristiana, in questa situazione sembra essere spenta, una buona dose di colpa è da attribuire a coloro che mantengono questo stato nella chiesa, $i$ due papi coi cardinali ${ }^{29}$. Per questi motivi, il Gelnhausen mette mano alla sua lettera. Ma c'è un altro ancora più importante, per cui l'intento "apologetico» è abbastanza chiaro ${ }^{30}$. L'expedit potest et debet concilium generale convocari sarà l'argomento del I capitolo. Questa conclusione principale viene sviluppata attraverso tre fondamentali ragioni. La prima è espressa con un sillogismo. La tesi: ciò che appartiene a cose minori, appartiene ancora di più alle cose maggiori. Per cui, se per cause minori si celebrarono concilii, quanto più ora, per la presente situazione. La

27 Il Gelnhausen inviò la stessa lettera al re di Francia Carlo V nel 1380 e nel 1381 al principe Ruperto, conte del Palatinato. Così le inscriptio sono due e contengono fondamentalmente la stessa idea espressa con parole non del tutto differenti.

28 Epistola, 111.

29 «Quem unus excommunicaverit, alius absolvit, quod unus relaxat, alter involvit; sicque pravis invalescentibus, bonisque deficientibus victor sedebit in solio maiestatis princeps tenebrarum artifex iniquitatis et circumquaque eriget tabernacula tyrannice potestatis», ibidem, 113.

30 «Porro quia post hoc vidi et relegi nonnulla opera et tractatus subtiles dominorum magnorum et peritorum valde, quorum calciamentorum eciam corrigias solvere non sum dignus», ibidem.

Proyección internacional de la iglesia hispana Hispania Sacra 51 (1999) 
maggiore è provata con alcuni episodi biblici e con canoni giuridici, mentre la minore è una lunga esposizione dei più importanti concilii dell'epoca apostolica e dei primi secoli della chiesa ${ }^{31}$. Gli apostoli potevano certamente risolvere qualsiasi problema attraverso lettere che pur scrivevano sotto l'ispirazione divina, eppure hanno voluto scegliere la via del «concilio» per dare l'esempio ai loro successori. Non si può tuttavia obiettare a questo, aggiunge il Gelnhausen, dicendo che la differenza numerica dei cristiani ora non permetterebbe un raduno generale, perchè non necessariamente tutti debbono partecipare, basta la rappresentanza dell'intera chiesa. Inoltre, l'esempio che viene dalla convocazione dei primi quattro concili ecumenici antichi è circa l'argomento che venne trattato: la fede. Ora: respondetur quod casus hodiernus est maximus casus fide $i^{32}$

La seconda ragione principale si ricollega alla precedente. La situazione creata dallo scisma perdura nella chiesa e dura a morire. Anzi, proprio coloro che dovrebbero porre fine ad esso fomentano e allargano sempre più questo male all'interno dell'intera chiesa ${ }^{33}$, per cui essi non possono garantire il ritorno della pace, essendo loro stessi causa di questo disordine. L'unica via possibile resta quella del concilio, come quando un concilio o sinodo diocesano viene convocato per risolvere i problemi della diocesi. Infine la terza ragione tocca punti più fondamentalmente ecclesiologici. Il concilio è l'organo che rappresenta l'universalità della chiesa ed è superiore allo stesso collegio cardi-

31 Per esempio cita quattro"concili" generali del periodo apostolico: il primo è in At 1, 20: il raduno dei discepoli con Maria in attesa dello Spirito; il secondo è in At 6, quando gli apostoli decidono di istituire i diaconi per il servizio delle mense; il terzo è il cosiddetto concilio di Gerusalemme di At 15; il quarto, infine, è in At 21, 18: Paolo espone a tutti i convocati ciò che Dio ha operato in mezzo ai pagani. Dei concili dei primi secoli richiama soprattutto i primi quattro ecumenici" Nicea del 325 , Costantinopoli del 381, Efeso del 431 e Calcedonia del 451.

32 Ibidem, 119. È interessante notare che il Gelnhausen vede lo scisma come problema di fede, che può essere causa di eresia nella chiesa. Per questo punto si ricollega, molto probabilmente, a tutta un'interpretazione giuridica che vede lo scisma nella chiesa come un'eresia sulla scia di Agostino. Per cui questo termine oltre ad avere un significato propriamente stricto, in senso lato comprende anche tutto ciò che potrebbe nuocere al bene della chiesa; in questo caso esso presenta la chiesa con una duplice capita quasi monstnum. In queste parole sembra riecheggiare l'Unam Sanctam di Bonifacio VII.

33 Lapidarie queste parole: «.,quamvis utraque pars legatos destinaverit, et ipsimet cardinales se diffuderint ad diversas regiones orbis, et extremum virium suarum extendesint, sicut terebinthus expandit ramos suos, predicando, scribendo, informando et alia multa faciendo, non tamen dictum \$cisma cessavit; immo procedit et crescit de malo in peius, et corda regum, principum et potentum de die in diem contra alterutrum videntur magis ac magis inflammari, et hereses maltiplicantur; quoniam numquam, vel raro scisma est sine heresi, eo quos scindens Ecclesie unitatem hereses sibi fingit, ut recte ab Ecclesia videatur recessisse», 120. II Gelnhausen descrive benissimo la situazione di quel breve periodo intercorso tra l'elezione di Clemente VII e il maggio del 1380. L'intento di dichiarare l'invalidità dell'elezione di Urbano VI e la legittimità di Clemente VII furono oggetti di trattati e di discorsi che si diffusero a macchia d'olio. Per esempio, si pensi al trattato del Flandrin. 
nalizio, che fa parte del concilio, ma non è superiore a esso. Anche per questa ragione esiste un sillogismo: la maggiore è chiaramente manifesta, perchè papa Niccolò I ( 858-867) riaffermò la supremazia del papato sull'intera chiesa ${ }^{34}$. La minore è che il collegio dei cardinali subsit alla chiesa, che il concilio generale rappresenta. Ora, se la salvezza non esiste fuori dalla chiesa è pur vero che la chiesa in cui si trova la salvezza è superiore a quella in cui non si ha salvezza. Per cui, se il collegio dei cardinali col papa devia in un crimine mortale non può più essere luogo di salvezza, e siccome la Chiesa universale non può mai deviare dalla fede, perchè ha come fondamento lo stesso Cristo, essa è superiore al collegio dei cardinali col papa. Il concilio che rappresenta l'intera chiesa è superiore al papa e al collegio cardinalizio che ha deviato nella fede ${ }^{35}$. Ma il papa può deviare dalla fede? Il Gelnhausen porta alcuni esempi storici come quello di papa Marcellino e di altri sostenendo giuridicamente col passo del Si papa: papa a nemine iudicari potest, nisi deprehendatur a fide devius ${ }^{36}$.

Nel capitolo II Gelnhausen espone molto brevemente le ragioni contrarie all'idea del concilio. Possiamo dire che le enuncia soltanto, perchè esporrà il suo pensiero ecclesiologico e giuridico successivamente:

1. «Quod semel est definitum per principem vel concilium, ultra ad nova concilia venire non sinit»

2. «In nulio notorio actus permanentis,quod excusacionem non admittit, est necessaria sentencia, et per consequens nec concilium, nec alius iudex quicumque»

3. «Impossibile est sine pape concilium generale fieri vel celebrari».

Ma prima ancora di rispondere a queste tre obiezioni che venivano mosse dalla parte contraria, il Gelnhausen nel terzo capitolo pone tre considerazioni fondamentali per far comprendere ancora più pienamente il suo pensiero ecclesiologico. Prima di tutto egli afferma che il capo indefettibile della chiesa è Cristo e non il collegio dei cardinali col papa ${ }^{37}$. Inoltre la Chiesa, che è con-

\footnotetext{
34 Nicolò I fu uno dei papi più importanti nel periodo che va da Gregorio Magno a Gregorio VII. Il suo pontificato, durato solo nove anni, riusci a far riacquistare alla $S$. Sede tutta quell'importanza che in seguito Gregorio VII riaffermerà e aumenterà.

35 Ė importante notare subito che la superiorità del concilio sul papa e sul collegio cardinalizio è ribadita dal Gelnhausen solo nel caso di eresia, di deviazione dalla vera fede. Non è dunque un assunto di ecclesiologia conciliare contrapposta a quella primaziale. Solo nel caso di eresia il concilio, espressione della chiesa universale, può garantire la vera fede.

36 Decretum Gratiani: D. XL. c.6: punto fondamentale per la giustificazione del concilio, come vedremo più avanti.

37 «Prima quidem, quod, sicut in deduccione tercie racionis principalis superius ad propositum ostendendum adducte tactum fuit, Ecclesia sancta catholica, cuius indefectibile caput est Christus... non est collegium pape et cardinalium, nec aliquod collegium particulare mundi", 128. A sostegno di
}

Proyección internacional de la iglesia hispana Hispania Sacra 51 (1999) 
gregata nella fede, speranza e carità dal Cristo, è amata da Lui e si presenta quale vergine casta davanti al suo Sposo ${ }^{38}$. La fede del Cristo è presente nell'intera chiesa che comprende anche il papa e solo questa fede può salvare il papa come qualsiasi altro fedele, uomo o donna che sia. Per cui, la comunità ecclesiale ha un duplice capo, uno principale, spirituale sempre sano e indefettibile: Cristo, l'altro secondario: il papa che è vicario del primo capo ${ }^{39}$. Essendo Cristo il capo della chiesa, egli è pure garanzia della sua indefettibilità nella fede, perchè dal Cristo la chiesa ottiene la pienezza di ogni grazia. Lui è il Verbo incarnato, unisce in sè l'umano e il divino, è il sacramento della fede. Il papa, invece, è capo secondario della chiesa e può essere anche manchevole nella fede senza l'assistenza divina.

La seconda considerazione mette in evidenza il bisogno della convocazione del concilio in casi estremi come il presente. Il Gelnhausen, con un gioco di parole, dice che tutto ciò che fuori del tempo di necessità è lecito compiere, nel tempo di necessità diventa debito, e ciò che fuori del tempo di necessità diventa illecito, nel tempo di necessità è debito. Così, il concilio dev'essere convocato per metter fine a questa situazione pericolosa per la vita della chiesa senza aspettare l'autorità per la convocazione ${ }^{40}$. Ora, ciò è possibile perchè non fa parte della ragione formale del concilio il fatto che debba essere convocato dal papa o per suo mandato, perchè se il papa occupa la sede pur essendo un eretico pertinace non ha nessuna autorità sulla chiesa. La stessa cosa varrebbe se un papa coi cardinali dichiarasse una sentenza dubbia in materia di fede, l'unico organo di vero giudizio sarebbe in questo caso il concilio, per il bene di tutta la chiesa. Per cui, il legislatore che fa le leggi non può assolutamente tenere presente tutti i casi possibili della sua applicazione, ma solo quelii che più solitamente si verificano. Ecco, l'ultima considerazione.

Finalmente arriva al quarto capitolo per rispondere direttamente alle obiezioni, di cui ha gia detto nel secondo capitolo. Prima di tutto deve rispondere alla dichiarazione che bisogna credere a ciò che i cardinali hanno detto e scritto. Il Gelnhausen non accetta naturalmente questa idea $e$ adduce un fatto fondamentale, prova infatti che le dichiarazioni dei cardinali sono notoriamente in contraddizione tra loro, al punto che alcuni affermano: A è papa, altri: A non è

questa affermazione fondamentale adduce l'autorità della Sacra Scrittura: $1 \mathrm{Cor} 11,3 ; \mathrm{Col} 1,18$; Os 2 , 20 e di alcuni passi patristici.

$38 \mathrm{Cfr} 2 \mathrm{Cor} 11,2$.

39 «Huius eciam alme matris universalis Ecclesie duo sunt vel esse debent capita subordinata spiritualia, unum quidem principale, semper sanum et indefectibile, quod est Christus Deus noster, vere fidei rector, quam sibi uni desponsatam et intactam servat virginem....aliud est caput Ecclesie secondarium, scilicet papa, qui est vicarius primi capitis, Christi», 129.

4 (i) Cfr 131 . 
papa $^{41}$. La soluzione dev'essere data dal concilio, in modo tale che si può conoscere a quale parte si deve credere:concilium de iure debeat in casu isto celebra$r^{42}$. Visto, dunque, che le dichiarazioni dei cardinali sono contraddittorie e non possono dare un'idea chiara della situazione, l'unica via è quella del concilio.

L'interesse del Gelnhausen in questa prima parte delle risposte è quello di screditare tutti i tentativi della parte opposta di dare importanza e soprattutto valore alle dichiarazioni dei cardinali. L'insostenibilità delle loro tesi rende chiaramente necessario il concilio. Proprio la categoria di necessità gli servirà per risolvere in termini giuridici il problema della convocazione del concilio senza l'autorità del papa. Cavallo di battaglia degli oppositori, la risposta a quest'obiezione occuperà la seconda parte di quest'ultimo capitolo. Mentre le altre risposte erano più a carattere disciplinare e di delucidazione, questa invece tocca il centro della questione. Il Gelnhausen deve dare la soluzione alla spinosa questione della impossibilità del concilio senza il papa. Dell'importanza di questa questione il preposto di Worms è cosciente: ad tercium, quod aliqui fortissimum esse censent...

La risposta si articola attraverso un'argomentazione logica che pone le premesse a soluzioni pratiche proposte dallo stesso autore per un eventuale concilio. Il Gelnhausen ritiene che il concilio si pud̀ convocare senza 1'autorità del papa in duplice modo. Nel primo caso una convocazione del concilio fatta senza l'autorità del papa concors et indubitatus Ecclesie presidens è da ritenersi nulla, per cui non si può parlare di un concilio, ma di un «conventiculum seu conciliabulum». Nel secondo caso, invece, può avvenire una convocazione conciliare per una causa razionale senza l'autorità del papa indubitatus per pura negligenza o per inerzia; oppure ci può essere una convocazione conciliare senza il consenso del papa o perchè nullo è il papa, e quindi vacante la sede apostolica, o perchè si hanno due papi indubbi; infine, o perchè pur essendoci concors papa, che non dà il suo consenso per la convocazione, la necessità razionale e urgente giustifica la convocazione senza il suo permesso ${ }^{43}$. Tutto questo dev'essere sostenuto ulteriormente da un altro elemento di carattere giuridico che faccia da base all'intera argomentazione. Ecco che Gelnhausen applica il principio dell'epicheia per interpretare quanto è stato stabilito dal legislatore ${ }^{44}$. L'autorità del concilio, logicamente, in questo caso verrebbe da Cristo stesso in quanto capo indefettibile della Chiesa.

Il principio dell'epicheia addotto dal Gelnhausen in quest'ultimissima parte della sua lettera si pone come unica ragione sufficiente per poter giustificare la

\footnotetext{
4] Cfr 133.

42 Ibidem.

43 Cfr le pagine 136-137.

44 Ibidem, 137.
}

Proyección internacional de la iglesia hispana Hispania Sacra 51 (1999) 
convocazione del concilio di fronte alla vigente legislazione ecclesiastica che contempla solo il papa come colui che ha il potere di convocare il concilio in via ordinaria. Non si tratta, così, di far saltare completamente l'ecclesiologia che vede il primato del papa una posizione importante nella Chiesa per poter affermare l'importanza e la superiorità del concilio sul papa, quanto invece di trovare una soluzione che potesse soddisfare non solo l'aspetto pratico della questione ma soprattutto quello teologico e giuridico. Quest'intuizione resta fondamentale, e tra coloro che hanno proposto il concilio in questo periodo, il Gelnhausen è conosciuto proprio per questa sua soluzione giuridica. L'epicheia viene applicata alla legge, come l'interpretazione serve per comprendere la parola del Cristo, come lui stesso afferma. Pur tuttavia, questo punto non è il cuore del trattato del Gelnhausen. Se ci si soffermasse solo a questo si farebbe un torto al suo autore. Il centro si deve trovare nel capitolo terzo, dove il preposto di Worms espone chiaramente la sua visione ecclesiologica, anzi senza questo capitolo non si comprenderebbe affatto la soluzione di carattere giuridico. Basterebbe notare la stessa posizione degli argomenti nei vari capitoli per comprendere questo punto. Le risposte alle obiezioni esposte nel capitolo secondo vengono date solo nel capitolo quarto, mentre il terzo serve come una premessa necessaria per comprendere il seguito.

L'Epistola concordiae non è solo uno scritto di carattere pratico. Presenta, invece, un'ecclesiologia propria in cui si sottolinea la derivazione della chiesa dal Cristo suo principale e indefettibile capo. Il papa, invece, è visto come capo secondario e inserito nella chiesa, non sopra di essa. Infine, il concilio è espressione dell'universalità della chiesa. Nessun altro organo potrà mai arrogarsi questa peculiarità. L'epistola, dunque, presenta una formulazione di carattere teologico molto più profonda del trattato dell'Ameilh. In quest'ultimo l'aspetto giuridico è molto più marcato e, inoltre, presenta un'ecclesiologia insostenibile, come più avanti vedremo. Questa differenza si nota anche dal maggior utilizzo della Sacra Scrittura da parte del Gelnhausen a differenza dell'Ameilh ${ }^{45}$. I richiami biblici si notano soprattutto nei capitoli in cui espone la dottrina ecclesiologica, mentre quasi per nulla si trovano nei capitoli in cui risponde alle obiezioni, proprio perchè i trattati degli oppositori al concilio avevano una consistenza particolarmente giuridica.

$45 \mathrm{Si}$ possono contare una sessantina di citazioni bibliche nel corso dei quattro capitoli. La maggior parte di esse sono del Nuovo Testamento, soprattutto i luoghi ecclesiologici delle lettere paoline. 


\section{CONCILIARISMO O ECCLESIOLOGIA CONCILIARE IN GELNHAUSEN?}

La contrapposizione del trattato del Gelnhausen con quello dell'Ameilh potrebbe facilmente farci deviare da una retta comprensione del pensiero del preposto di Worms. Leggendo e analizzando la Epistola concordiae accanto al Contra petentes dell'Ameilh, un giudizio affrettato potrebbe correre il rischio di classificare il Gelnhausen come colui che si schiera per il concilio e per questo potrebbe essere inserito nella schiera di coloro che secondo la tradizione entrano a far parte del pensiero cosiddetto conciliarista che sfocerà nel conciliarismo ${ }^{46}$, e l'Ameilh, invece, sarebbe il rappresentante dell'ecclesiologia primaziale, secondo cui il papa non potrebbe mai essere giudicato da un concilio come sembra trovarsi in coloro che auspicano tale celebrazione. In verità analizzando attentamente lo scritto del Gelnhausen si ci trova davanti ad un autore che raccoglie il pensiero ecclesiologico precedente e tenta di dare una soluzione concreta al problema posto in questione: convocare il concilio è possibile o meno? Proprio su questo punto egli entra in quella corrente di pensiero che affonda le sue radici tra il XII e XIII secolo, ma che proprio nel XIV secolo trova la massima espressione speculativa, che più tardi sarà vista come prodomo del conciliarismo se non addirittura fondamento della stessa teoria conciliare. Eppure un giudizio del genere sarebbe del tutto affrettato per non dire alquanto impreciso. La corrente del pensiero conciliare così come si presenta nei vari autori del XIV secolo non è del tutto univoca, nè tanto meno in

46 Sul conciliarismo la bibliografia è abbastanza notevole. Pur tuttavia, una scelta necessaria è da fare su una base precisa che offra la possibilità di avere una visione generale del problema, ma anche una più precisa analisi della questione. Per cui ci siamo rivolti a scritti ben precisi: il primo non poteva non essere che il libro del B.TıERnEY, Foundations of the Conciliar Theory, Cambridge 1955 (ripr.1968), che resta uno dei lavori più precisi e approfonditi su questo tema. Il contributo originale è stato quello di chiarire l'origine della teoria conciliare nell'ecclesiologia del XII e XIII e non nel secolo XIV come spesso si è detto. Inoltre, l'articolo di P. BARBAINI, Per una storia integrale delle dotrine conciliari, in La Scuola Cattolica 89 (1961), 186-204; 243-266, che offre una visione panoramica della dottrina conciliare ponendo l'accento su alcuni dei più importanti rappresentanti di questa corrente. A.C. LEOPARDI, Il conciliarismo: genesi e sviluppo, Bari 1978, che studia il problema in modo evolutivo facendo vedere come tra il XIV e XV secolo la corrente dottrinale assume spessori più marcatamente concreti. G.ALBERIGO, Chiesa conciliare. Identità e significato del conciliarismo, Brescia 1981. In questo libro l'autore fa notare come l'idea del conciliarismo si è presentata durante la celebrazione dei concili di Pisa, Costanza e Basilea, con una particolare attenzione al decreto dell'Haec Sancta di Costanza e í problemi annessi. AA.VV., Conciliarismo, Stati nazionali, inizi dell'umanesimo,Atti del XXV Convegno storico intemazionale del Centro di studi sulja spiritualità medioevale (Todi, 9-12 ottobre 1988), Spoleto 1990. Non poteva mancare H. JEDIN, Storia del Concilio di Trento, vol. I, Brescia ${ }^{3} 1987,13-157$, in cui esamina la corrente della dottrina conciliare nel secolo XV e nota come essa si trasformerà in conciliarismo e provocherà la reazione del papato con la restaurazione dell'autorità papale. Infine per una visione generale, Y. CONGAR, L'Eglise de Saint Augustin à l'èpoque moderne, Paris 1970, soprattutto il capitolo X.

Proyección internacional de la iglesia hispana Hispania Sacra 51 (1999) 
coloro che hanno scritto a favore del concilio durante lo scisma occidentale ${ }^{47}$. Una facile generalizzazione potrebbe farci inserire in un unico gruppo questi pensatori e affermare che si tratta di un'unica questione: il concilio è superiore al papa e coloro che durante lo scisma hanno chiesto la celebrazione del concilio sarebbero rappresentanti se non addirittura i promotori concreti di quest'idea ecclesiologica, contro cui i sostenitori della vera ecclesiologia primaziale hanno combattuto tenendo fermo il principio teologico e giuridico dell'impossibilità di giudizio della sede di Pietro.

Non ci troviamo affatto davanti a questa situazione, perchè sotto il concetto di concilio, conciliarismo ed ecclesiologia primaziale si nasconde una riflessione che ha richiesto tempo e fatica per avere una retta idea della chiesa o per una travisazione che vedrà alcune conseguenze anche pratiche. Il giusto posto che il concilio deve avere all'interno della chiesa sarà un problema che proprio in questo secolo avrà richiesto una precisa posizione dottrinale che salvaguardasse la stessa autorità del papa su tutta la chiesa.

È necessario, dunque, fare una debita precisazione senza presumere di dire qualcosa di nuovo sul concetto di concilio così come è venuto a precisarsi in alcuni esponenti di questa corrente durante il secolo XIV, per comprendere meglio il contributo del Gelnhausen, le sue dipendenze da questi ma anche le sue differenze che sono notevoli.

\subsection{Una radice del conciliarismo}

Il Decretum Gratiani, che come sappiamo è una raccolta di leggi, scritti di papi e vescovi, atti di concili e sinodi su questioni disciplinari e giuridiche, afferma che la sede di Pietro, la prima sede, non può essere giudicata da nessuno ${ }^{48}$. L'autorità del successore di Pietro non può essere sottoposta a qualsiasi giudizio da parte di nessuno anche se si trattasse dell'imperatore o di tutto

47 Nel periodo propriamente dello scisma oltre al Gelnhausen e al Langenstein, di cui già abbiamo detto, hanno scritto sul concilio altri teologi e giuristi: PIERRE D'AlLLY (1340-1420) col suo trattato si pone sulla linea di Ockham; FRANCESCO ZABARELLA (1360-1417) con Tractatus de schismate sui temporis fonda insieme i due elementi della dottrina conciliare: rappresentatività e corporatività accompagnati da elementi propri del principio democratico; JEAN GERSON (1363-1429), teologo molto più complesso dei precedenti, svihuppò la sua ecclesiologia in due periodi divisi dal concilio di Pisa (1409). Nel secondo periodo il suo pensiero diventa più maturo, espressione ne è il De potestate ecclesiastica; DIETRICH VON NIEM (1340-1418) con De modi uniendi Ecclesiam presenta un'idea più estremista di tutti.

48 Causa IX, q.3, cap.XIU: Prima Sedes. «Nemo iudicabit primam sedem iustitiam a temperare desiderantem. Neque enim ab Augusto, neque ab omni clero, neque a regibus, neque a populo iudex iudicabiturn. 
il clero messo insieme. La ragione fondamentale di questo principio si trova nel fatto che giudicare diventa ipso facto sinonimo di superiorità sull'altro, per cui giudicare la sede romana potrebbe significare superiorità sul papa, il che è inammissibile. La coscienza di questa superiorità del papa su tutta la chiesa è presente chiaramente nell'espressione di questo principio, per cui salvaguarda l'idea fondamentale della funzione del papa e della sua piena autorità all'interno della chiesa. Il suo servizio è la conferma nella fede dei credenti attraverso l'esercizio del ministero dell'insegnamento che è garanzia dell'autenticità della fede cattolica. Tuttavia, se proprio nella fede il papa dovesse deviare, allora lo stesso Decretum prevede che esso può essere sottoposto a giudizio senza specificare da parte di chi $^{49}$. Quale organo può e ha all'interno della chiesa l'autorità di giudicare il papa deviato dalla retta fede ed essere così garante di quella vera, compito proprio del pontefice?

Quest'eccezione ha posto le fondamenta per il conciliarismo. La questione del «de Pontefice haeretico» ha «scatenato» una vera e propria lotta di trattati ecclesiologici da parte di teologi e giuristi che cercarono di trovare la soluzione al problema enunciato ma non risolto da Graziano. Eppure una corrente si fa sempre più avanti ed è quella che vede il concilio come unico organo che può giudicare il papa, in quanto esso rappresenta l'intera chiesa o perchè esso riprende nelle proprie mani il potere che è stato delegato ad alcuni, come allo stesso papa. Al di sotto di questa duplice idea sussistono due concezioni ecclesiologiche, che in modo più o meno accentuate si ritrovano in quasi tutti $\mathrm{i}$ rappresentanti della dottrina conciliare: concetto di rappresentatività e quello di corporatività. L'idea di rappresentatività sussiste nel fatto che il concilio rappresenta l'intera chiesa, e così ottiene l'autorità dalla stessa chiesa che deve rappresentare nell'assemblea conciliare. L'altra idea, invece, ancora più fortemente, ritiene che l'autorità è e resta in fondamento nella chiesa, ma il suo esercizio viene demandato in mano a uno $o$ a più, ma la chiesa ha sempre la possibilità di riprenderlo qualora questo o questi risultassero inetti a tale compito. Dunque, come il potere della chiesa locale è in mano al collegio dei canonici e viene demandato al vescovo, così quello del papa è in mano a quello del collegio cardinalizio che rappresenta l'intera chiesa. Sembra che proprio quest'ultima idea abbia maggiormente influito sul concetto del conciliarismo ${ }^{50}$.

49 Dist.XL, cap.6: Si Papa: «Si Papa suae, et fraternae salutis negligens, deprehenditur inutilis, et reuissus in operibus suis, et insuper a bono taciturnus, quod magis officit sibi, et omnibus, nihillominus innumerabiles populos catenatim secum ducit primo mancipio gehennae, cum ipso plagis multis in aeternum vapulaturus. Huius culpis istic redardequere prebumit mortalium nullus: quia cunctos ipse iudicaturus a nemine est iudicandus, nisi deprehendatur a fide devius: pro cuius perpetuo statu universitas fidelium tanto instantibus orat, quanto suam salutem post Deum ex illius incolumitate animadvertit propuesinus pendere».

so Cfr B. TIERNEY, op. cit., 132-153.

Proyección internacional de la iglesia hispana Hispanja Sacra 51 (1999) 


\subsubsection{Prima del Gelnhausen}

Il primo rappresentante di questa corrente è possibile riscontrarlo in Giovanni Quidort, più comunemente conosciuto come Giovanni da Parigi, che proprio durante la lotta tra Bonifacio VIII e Filippo il Bello scrisse il suo De potestate regia et papali $(1302-1303)^{51}$. Si muove nel campo dell'ecclesiologia che oscilla tra il concetto di rappresentatività e quello di corporatività tanto da vedere nella congregatio fidelium l'origine dell'autorità che deriva direttamente da Dio. Il papa, in questa visione, non è altro che il dispensator dei beni spirituali e temporali. Egli ha un'autorità che non può essere intesa in senso assoluto perchè soprattutto in questioni di fede è soggetto al concilio, quindi non può stabilire nulla che possa essere contro lo stesso concilio. Da notare però che non ci troviamo davanti ad una estremizzazione dell'idea conciliare. Giovanni da Parigi intende ancora il concilio col papa e non senza o contro di esso, pur tuttavia la sua plenitudo potestatis ha subito un duro colpo ${ }^{52}$. Inoltre, il concilio proprio in funzione dell'autorità che viene dall'intera chiesa può deporre il papa, in quanto riprende nelle proprie mani il potere dato al papa come delegato. Le idee esposte da Giovanni Quidort sembrano collimare perfettamente con l'idea giurisdizionale del potere del papa. Il tentativo di ridimensionare la sua giurisdizione si cerca di trovarlo in un'idea ecclesiologica che vuole salvaguardare il principio della collettività della chiesa dal dispotismo papale per poi dare spazio a quello regale. Non a caso il tentativo ancora più estremizzato sarà dato da Guglielmo Durante $(+1328)$ nel suo Tractatus de modo concilii generali celebrandi scritto su invito di Clemente $\mathrm{V}$ per il concilio di Vienne ${ }^{53}$. Il papa è visto nella visione ciprianea come il «primus inter pares» ed è soggetto al concilio che è un' autorità accanto al papa se non addi-

51 Cfr ibidem, 157-178, inoltre J. LECLERQ, Jean de Paris et l'Ecclesiologie du XIII siècle, Paris 1942. Non a caso iniziano con questo autore. L'invocazione del concilio da parte dei cardinali Colonna contro il papa per la questione della rinuncia del papa Celestino V e la nomina di Bonifacio VIII a papa si intreccia pienamente con la lotta che questo papa fece con il re di Francia per la libertas ecclesiae. Il concilio veniv'a invocato come la possibilità di ridimensionare il potere del papa motivandolo con questioni di tipo spirituale: il papa sarebbe un tiranno, accentratore e avrebbe fatto dimettere Celestino per poter salire al soglio pontificio. Tuttavia i cardinali Colonna sono sostenitori dell'idea primaziale della chiesa. Il concilio è invocato per l'abdicazione di papa Celestino e per la nomina di Bonifacio che secondo loro resta illegittima, in quanto non poteva avvenire l'abdicazione.

52 Cfr P. BARBAlN1, ant. cit., 194-195.

53 Interessante notare come anche quest'autore cerca di ridimensionare la potestas del papa in nome dell'universalità della chiesa che si esprime nel concilio. Non a caso il concilio di Vienne, convocato da Clemente $\mathrm{V}$ per questioni varie e più volte richiesto da Filippo il Bello per condannare Bonifacio VIII, sarà la possibilità concreta nelle mani del re dei francesi di liberarsi dei Templari. Ci troviamo ancora una volta davanti ad una chiara tendenza di ridimensionamento della plenitudo potestatis del papa per affermare quella del concilio, facile strumento nelle mani del potere civile per propri interessi. 
rittura superiore a lui. Non può decidere senza il concilio e non può assolutamente abrogare cambiare riformare ciò che è stato deciso dal concilio. Questi, infatti, rappresenta l'intera chiesa e le sue leggi riguardano tutti, per cui ciò che si decide dev"essere deciso da tutti, ciò che può fare solo il concilio. Alla base di quest'idea sussiste il principio del Quod omnes tangit ab omnibus approbari debet che accompagnerà quasi tutti coloro che troveranno nel concilio l'espressione della democrazia nella chiesa ${ }^{54}$. Di questa poi si farà promotore particolarmente Marsilio da Padova (+1342) nel suo Defensor pacis. Massimo rappresentante della corrente conciliarista, è stato uno di quelli che ha dato più impulso allo sviluppo del conciliarismo posteriore. La chiesa è vista da Marsilio all'interno dello stato e sottomessa a esso perchè la universitas civium delega ad alcuni uomini l'esercizio del potere e dell'autorità. Così il concilio è la massima espressione della chiesa e unico organo autoritativo nella stessa che è vista all'interno dello stesso stato. Il potere così deriva dai cives che formano lo stato e che delegano tale petere al principe per la parte temporale e al concilio per ciò che è spirituale, ma tutto è visto in un orizzonte in cui il «cesaropapismo» con base democratica deve togliere ogni potere al papa ${ }^{55}$. Anzi, Marsilio nega qualsiasi fondamento scritturistico e dommatico del primato petrino che non è altro che il risultato di vicende politiche e sociali. In quest'autore troviamo il rappresentante più autorevole della tendenza di questo secolo che vede intimamente connesse vicende politiche e tentativi dottrinali che cercano di replicare all'ecclesiologia medioevale con una nuova che salvaguardi gli interessi del principe.

Da questa visione così estrema si distanzia chiaramente Guglielmo di Ockham $(+1359)$ nel suo Dialogus $5^{56}$, Breviloquium de potestate papae ${ }^{57}$. Come prima cosa afferma l'origine divina del papato e quindi la sua funzione insostituibile all'interno della chiesa. Non presenta una visione estrema come quella di Marsilio da Padova, ma alquanto equilibrata. Il papa come anche il concilio servono alla vita della chiesa ed hanno un loro posto ben preciso. La chiesa non è per Ockham personificata nel papa nè tanto meno nel concilio, ma essa è la

54 Per questo punto e l'applicazione di questo principio cfr l'articolo di A. MaRONGIU, $I l$ principio della democrazia $e$ del consenso (Quod omnes tangit, ab omnibus approbari debet) nel XIV secolo, in Studia Gratiana 8 (1962) 553-575.

55 Con Marsilio da Padova ci troviamo davanti al secondo grande conflitto tra il papa e 1'imperatore. In ogni caso il problema resta lo stesso: la fonte del potere temporale deriva da quello spirituale oppure direttamente da Dio nel popolo? Marsilio parteggia per questa seconda linea, per cui le sue idee influenzeranno talmente Ludovico il Bavaro che l'incoronazione imperiale avverrà nel 1328 per le mani del capitano del popolo in Roma anche se dopo la consacrazione da parte di due vescovi. Ci troviamo davanti ad una vera e propria incoronazione laica dell'imperatore.

s6 II Dialogus in M. GoLDAST, Monarchia S. Romani Imperii, t. II, Francoforte 1614, $394-957$.

57 Ed. crit. di L. BAUDRY, Paris 1937.

Proyección internacional de la iglesia hispana Hispania Sacra 51 (1999) 
congregatio fidelium, in cui la presenza continua del Signore è garanzia della sua indefettibilità. Questa fede può trovarsi anche soltanto in una donna contro l'intera chiesa, ciò è segno della presenza del Cristo che garantisce l'immunità della chiesa dall'errore nella fede. Questo elemento è proprio della chiesa e non del concilio che come il papa può errare. Pur tuttavia nel caso che si avesse un papa eretico, questi dev'essere sottoposto a giudizio o da quello ortodosso o dal concilio o dallo stesso collegio cardinalizio. In Ockham non troviamo una teoria esplicitamente conciliarista. Anche se quest'autore ha maggiomente influito sugli scrittori del periodo dello scisma non può essere considerato un conciliarista, o almeno un estremista in questo campo. La sua resta una precisazione dottrinale più che giuridica. L'elemento teologico diventa più preponderante di quello giuridico, dove la questione della giurisdizione e della potestà si fa più fortemente presente. Ecco perchè si situa in maniera equilibrata a differenza di quelle idee sorte per contrasti politici.

L'elemento dominante in questi principali pensatori resta, dunque, quello della superiorità in certo senso del concilio sul papa. L'idea dell'infallibilità della chiesa applicata al concilio come rappresentante dell'intero corpo permane all'interno di queste proposte più o meno accentuate in campo teologico. Il bisogno di trovare una soluzione al problema giurisdizionale e alla potestà del pontefice romano accresce tutte quelle premesse teologiche e giuridiche che nel medioevo erano andate sviluppandosi. Così il concilio diventa un'arma nelle mani dell'intera chiesa per poter mettere fine al potere dispotico del pontefice. Se tutti questi tentativi sorgono in momenti chiaramente conflittuali, pur tuttavia essi hanno dato, ad eccezione di qualcuno, un contributo per poter chiarire la questione della giudicabilità del papa, nel caso che questi cadesse in eresia. Il lavoro dei pensatori durante lo scisma d'occidente fu di poter giustificare la convocazione del concilio senza il papa per una questione che riguardava l'intera chiesa. In essi non si trova un conciliarismo estremo, ma solo il tentativo di comprendere dottrinalmente la natura della chiesa e salvagurdare la funzione del suo capo visibile: il papa. Stranamente non si è posto l'accento proprio su questo punto. I fautori del concilio, in questo arco di tempo, hanno giustificato la sua convocazione proprio per riavere all'interno della chiesa il centro visibile della sua unità, il papa, cosa che il conciliarismo metteva in second'ordine rispetto al concilio stesso. Il concilio doveva essere solo lo strumento adatto per questo fine. Ma un'idea propriamente ecclesiologica comprendeva il posto dovuto al concilio come organo garante della vita dell'intera chiesa in quella determinata situazione. 


\subsubsection{La posizione del Gelnhausen}

Lo scritto del preposto di Worms si colloca a pieno titolo nel «mare magnum» delle pubblicazioni ecclesiologiche nel periodo dello scisma. Le sue due Epistolae sono il segno dell'interesse non solo per l'aspetto pratico, ma anche teologico delle questioni che si ponevano e delle soluzioni che si cercavano affannosamente. Egli è stato uno dei primi che hanno scritto sulla necessità del concilio per porre rimedio allo scandalo della divisione nella chiesa, e sollecitato anche da una diversa impostazione ecclesiologica approfondi ulteriormente il suo pensiero a riguardo del rapporto tra papa e concilio. Una cosa fondamentale interessa il Gelnhausen: giustificare il concilio. La sua lettera potrebbe essere letta a partire dalla duplice indicazione verbale: «potest et debet concilium generale convocari». L'autore elabora il suo scritto a partire da questi due verbi, per cui deve dimostrare che il concilio si può e che si deve convocare. In ogni caso, si tratta della giustificazione e della indispensabilità del concilio.

Per comprendere il pensiero ecclesiologico del Genhausen bisogna rifarsi subito al capitolo III della sua Epistola. In base a questa parte è possibile comprendere tutto il suo scritto e l'idea di fondo che permea l'intera questione dottrinale. Da questo stesso capitolo si può inoltre comprendere la sue dipendenze dagli autori precedenti, ma anche le sue distanze. Gelnhausen risulta un pensatore che rielabora il pensiero giuridico-teologico applicandolo alla sua determinata situazione che non sembra avere altri precedenti, per cui il compito è ancora più arduo.

\section{a) Elementi di derivazione ockhamista.}

La chiarezza della sua descrizione ecclesiologica è ammirevole. Gelnhausen non si perde in una lunga argomentazione sulla natura della chiesa e sulla potestà del papa, ma in modo molto breve e nello stesso tempo preciso espone la sua ecclesiologia. La Chiesa, per Gelnhausen, gode di una indefettibilità nella fede per mezzo di Gesù Cristo che è il vero capo della Chiesa. Questa indefettibilità è dovuta al fatto che la Chiesa è il corpo di Cristo, fatto suo grazie al rapporto quasi nuziale come in molte parti della Scrittura è dato leggere. Essa si fonda su Cristo e la fede in Lui è garanzia dell'appartenenza alla chiesa. L'indefettibilità nella fede non può mai venir meno, in quanto può sussistere anche in un solo uomo cristiano anche se non appartiene alla gerarchia. Proprio per questa fede indefettibile diventa nello stesso momento più grande di coloro che hanno errato nella fede ${ }^{58}$, anche se si trattasse del papa e dell'intero

58 A questo proposito, il Gelnhausen riporta l'evento della morte del Cristo e l'abbandono da parte dei suoi discepoli, mentre la sua Madre resta ai piedi della croce: «Hec itaque sancta mater

Proyección internacional de la iglesia hispana Hispania Sacra 51 (1999) 
collegio cardinalizio. Capo principale e spirituale è, dunque, Cristo, origine e fondamento dell'indefettibilità nella fede. La chiesa, però, essendo comunità visibile, ha pure un capo secondario e visibile che è il papa. Questi, per il Gelnhausen, è capo in quanto è vicario del vero capo che è Cristo e in quanto è fondato su di Lui che è capo; per cui Pietro, primo papa, deriva da Cefa che significa capo. In questa visione sussiste la cosiddetta esegesi africana (Cipriano e Agostino) del passo biblico di Mt 16, 18-19, per cui pietra sarebbe Cristo e così Pietro deriva da pietra e non pietra da Pietro. Così facendo il fondamento dell'intera autorità è data da Cristo all'intero corpo episcopale e non solo a una determinata persona come l'interpretazione che fa derivare Pietro da pietra fa intendere. Il fondamento del papato su Cristo fa sì che esso non muoia mai anche quando il papa venisse meno o cadesse in eresia, per cui è come se fosse morto ${ }^{59}$.

L'intera chiesa è rappresentata dal concilio. Questo è l'organo in cui l'universalità dei fedeli viene in modo del tutto eminente rappresentata. Contro l'idea che la chiesa Romana rappresenti l'intera chiesa, Gelnhausen pone il concilio come organo visibile della sua rappresentatività. In questo il preposto di Worms segue l'idea fondamentale di coloro che vedono il concilio secondo l'ottica della funzione rappresentativa. Non troviamo in lui il concetto della corporatività e neppure quello che vedeva nel concilio l'indefettibilità. Sembra che Gelnhausen non ammetta esplicitamente l'infallibilità del concilio, mentre parla chiaramente della possibilità che il papa e il collegio cardinalizio possono cadere in errore, non dice al contrario che il concilio gode di indefettibilità ${ }^{60}$. In questo segue I'idea di Ockham. Esso si convoca per questioni che ri-

Ecclesia non excludit, sed pocius includit papam, et queralibet fidelem, immo et in solo papa salvari posset, sicut et in quolibet alio fideli viro vel femina; unde fides Christi, cum omnes discipuli eo relicto fugerunt, M(a)r[ci] XIIII., in sola virgine Maria salva creditur remansisse; nam et ius universitatis in uno salvari potest, ut no[tatur] de postu. prela. c. Gratum», Epistola, 129.

$59 \mathrm{Si}$ nota un'analogia forte con Ockham in questo passo del Dialogus: «DISCIP. Qualiter respondetur ad motiua opinantium praedictorum, quibus probare nituntur, quod solus Christus erat fundamennum ecclesiae. MAGISTER. Ad primum eorum, cum adducitur Glossa interlinearis, qua probatur, quod Christus sibi videtur retinuisse fundamenti dignitatem. Respondetur per distinctionem de fundamento quia quoddam est fundamentum ecclesiae primarium et principale, absque quo nulla potest fundari ecclesia. Et illud fundamentum est sol (!) Deus sive Christus. Aliud est fundamentum ecclesiae secundarium, sine quo poterat fundari ecclesia, sine qua tamen non fuit fundata de facto, et tale fundamentum non est solus Deus nec solus Petrus: imo omnes apostoli sic fuerunt fundamenta ecclesiae, inter quos tamen quodammodo principalius et universalius fundamentum fuit beatus Petrus», in op. cit., 862 .

60 È da tener presente chiaramente la sottile differenza nella visione del Gelnhausen. Il concilio e superiore al collegio dei cardinali perchè questo pud errare, ma non dice esplicitamente che il concilio non pud errare. E un silenzio particolarmente loquace. In ogni caso, il Gelnhausen tiene presente quanto ha scritto Ockham: «..quod pie tenendum est interpretationes concilii generalis esse nobis reuelatus ab eodem spiritu, a quo reuelatae sunt Diuinae Scripturae. Respondetur, quod non est ne- 
guardano l'intera chiesa e queste devono essere trattate da tutti. Riemerge l'idea espressa dal principio del quod omnes tangit ab omnibus tractari debet. La situazione provocata dallo scisma è una situazione che riguarda l'intera chiesa.

In queste idee ecclesiologiche, Gelnhausen rispecchia chiaramente l'impostazione di Ockham. Tuttavia, un problema completamente nuovo urge allo scrittore per trovare una soluzione particolarmente chiara e per evitare critiche di carattere particolarmente giuridico. Egli deve risolvere il problema della convocazione del concilio senza il papa. Non è possibile un concilio senza il capo supremo o addirittura contro di lui. Allora l'unica possibile strada da percorrere è quella di trovare una soluzione giuridica valida. La novità del Gelnhausen sarà proprio in questo punto. Egli troverà nell'epicheia un statuto giuridico per poter giustificare il concilio senza il papa.

\section{b) Elementi differenti.}

La situazione completamente nuova in cui si trova il Gelnhausen, come tutti gli altri scrittori in questo periodo, è quella di dover giustificare il concilio per decidere chi dei due papi sia quello legittimo. Non gli interessa tanto stabilire una nuova dottrina sul concilio come se questo fosse fonte di un'autorità in campo dottrinale anche senza il papa, ma solo di poter risolvere una situazione ben precisa. Il problema della contesa dei due pretendenti alla sede apostolica non aveva altra via d'uscita se non quella del concilio quale organo neutrale per $i$ due.

La particolarità dello scisma fà si che il preposto di Worms si trovi in una situazione completamente differente da quella degli autori della prima parte del secolo XIV. Per questi, la dottrina sul concilio, a differenza di qualcuno più estremista, veniva sviluppata evidenziandone la possibilità $\mathrm{e}$ il suo statuto giuridico all'interno della chiesa anche quando si trattava di dover giudicare il papa, nel caso in cui questi fosse caduto in errore. Era chiaro, dunque, secondo la dottrina conciliare che l'assemblea del concilio poteva assumersi il compito di «giudicare» il papa qualora questi fosse deviato nella fede oppure il suo comportamento scandaloso facesse da ostacolo alla fede della chiesa. Il problema del Gelnhausen è completamente nuovo. Non si erano avuti altri precedenti nel corso della storia della chiesa. L'unico elemento comune con tutta la dottrina conciliare per questo caso era quello che si rifaceva alla questione del Si Papa presente nel Decretum Gratiani. Eppure qui non si tratta di dover giudicare un papa per eresia. La questione principale è quella di stabilire chi

cesse, nec semper oportet omnes Christianos hoc credere, quia concilium generale saepe innititur vel inniti potest sapientiae humanae: quae deuiare potest a vero", Dialogus, in op. cit., 824.

Proyección internacional de la iglesia hispana Hispania Sacra 51 (1999) 
dei due sia il vero papa. Ma il Gelnhausen utilizza questo stesso passo del $D e$ cretum per il suo scopo, perchè l'unico argomento giuridico valido è quello del «de Pontefice haeretico».

Dal I capitolo e dall'incipit della sua Epistola è possibile rilevare che per il Gelnhausen lo scisma con tutto ciò che comportò nella chiesa equivarrebbe ad una situazione di errore all'intemo del corpo ecclesiale. La vita della chiesa e la sua funzione di salvezza vengono meno. Per il Gelnhausen lo scisma presenta la Chiesa come un corpo mostruoso al mondo, mentre dovrebbe essere segno di un tutto organico. Non si tratta dunque di un'eresia vera e propria da combattere. I due papi non hanno aderito ad una qualsiasi eresia già condannata dalla chiesa, nè tanto meno hanno dato vita ad una nuova, ma la loro stessa presenza è uno scandalo all'interno e all'esterno della chiesa. Il motivo per giudicare i due papi è più che sufficiente. In questo senso il Gelnhausen interpreta la questione del nisi deprehendatur a fide devius in senso più generale: per fide devius si intende tutto ciò che può concretamente nuocere la chiesa. Per questo lo scisma è un problema di fede. Il Gelnhausen inequivocabilmente ritiene che la situazione della divisione è questione che tocca la chiesa, perchè riguarda il suo capo visibile. Così come gli altri concili sono stati convocati per chiarire questioni dottrinali, anche per questo caso esso deve servire alla sopravvivenza della chiesa e della fede. Se la convocazione del concilio è chiaramente giustificata per questi problemi, tuttavia Gelnhausen non si ferma a questo. Approfondisce ulteriormente la questione applicando lo statuto giuridico dell'epicheia.

Uno dei punti più geniali del pensiero del Gelnhausen è dato proprio da questa felice idea. La difficoltà insormontabile del problema della legittimità del concilio senza il papa è risolto, almeno in linea di principio, da questa applicazione giuridica. Il Gelnhausen spiega che applicando la legge al caso particolare, ciò necessita sempre una comprensione dello spirito della legge e della mente del legislatore. Proprio perchè si è presentata una questione completamente nuova e non prevista da nessun decreto o legge, si deve applicare la legge tenendo conto della situazione per cui essa serve. L'epicheia potrebbe bastare; eppure, Gelnhausen vuole ancora di più salvaguardare il principio per cui il concilio si celebra con la presenza del papa. Alla convocazione del concilio debbono acconsentire i due papi ${ }^{61}$. Esso non dovrà avere il compito di stabilire qualcosa di nuovo, ma solo quello di stabilire la verità riguardo ai due pretendenti. Fatto questo il vero papa accede al concilio e conferma quanto deciso.

Le idee espresse nel quarto capitolo del suo scritto non sembrano essere solo una risposta alle questoni prettamente giuridiche. Non si può vedere solo

61 Cfr p. 138. 
il terzo capitolo come sezione ecclesiologica e il quarto come risposte a difficoltà giuridiche. Il terzo capitolo si completa nel quarto. L'idea ecclesiologica del Gelnhuasen è presente tanto nel terzo quanto nel quarto capitolo. Anzi proprio in quest'ultimo è possibile comprendere che non ci troviamo davanti ad una tendenza propriamente conciliarista quanto ad una ecclesiologia conciliare. In Gelnhausen non troviamo un conciliarismo estremizzato. L'autore non si ricollega tourt-court alla linea dei precedenti scrittori di trattati ecclesiologici, ma prende solo alcune idee fondamentali per poter giustificare la sua richiesta. Una di queste è quella della rappresentatività del concilio ${ }^{62}$. In questo possiamo dire che il preposto di Worms si ricollega alla linea propriamente conciliare. Nel concetto di rappresentatività si trova la chiave di lettura dell'idea conciliare. Se la Chiesa intera gode dell'indefettibilità nella fede per la presenza costante dell'azione del Cristo capo principale, il concilio organo rappresentativo dell'universalita della chiesa partecipa di questa stessa indefettibilità, che non risiede nella membra dei fedeli ma nel Cristo stesso dal quale il concilio prende autorità se convocato senza il papa ${ }^{63}$. Esso è superiore al papa e al collegio dei cardinali, in quanto questi possono deviare nella fede, mentre ciò è impossibile per la Chiesa universale. Il concilio, dunque, è superiore al papa? Sul papa eretico certamente sì, ma non è principio basilare dell'ecclesiologia. Il Gelnhausen non può essere annoverato tra coloro che hanno posto tutte le fondamenta dottrinali al conciliarismo. Lo scopo del suo trattato è un altro. Il contributo principale è stato quello di distinguere chiaramente la Chiesa universale dalla Chiesa Romana. Ciò si comprende proprio in ragione al fatto che 1'Epistola concordiae fu scritta tenendo presente il trattato dell'Ameilh ${ }^{64}$. Il preposto di Worms rifiuta la soluzione proposta dall'Ameilh perchè rifiuta la sua ecclesiologia, in cui la chiesa universale veniva a identificarsi con quella romana.

\footnotetext{
62 «Sed sancta mater Ecclesia catholica et universalis, cuius concilium generale est representativum, est superior collegio cardinalium, quorum factum, fidem et tocius Ecclesie statum tangens, in dubium et questionem vertitur, nec est in terris aliquis alius superior, ad quem in hoc casu haberi valeat recursus», p. 122.

63 «Et si dicatur: cuius auctoritate concilium in tali casu censebitur congregari et acta in eo valitura: respondetur, quod auctoritate capitis indefectibilis et semper influentis Jesu Christi...», p. 136.

64 Il capitolo secondo dell' Epistola richiama le tesi fondamentali dell'Ameilh per poi rispondere nel quarto ed ultimo capitolo. Da ciò sì puó comprendere che l'intero scritto del Gelnhausen ha di mira due cose: da una parte rispondere alle obiezioni dell'Ameilh per una convocazione del concilio senza il papa, e queste sono di carattere prettamente giuridico, e dall'altra distinguere la Chiesa universale da quella Romana, questo a carattere prettamente teologico, proprio contro l'idea ecclesiologica dell'Embrunensis.
}

Proyección internacional de la iglesia hispana Hispania Sacra 51 (1999) 


\section{ECCLESIOLOGIA PRIMAZIALE O OLIGARCHICA IN AMEILH?}

Lo scritto del cardinal Ameilh, una volta letto l'Epistola brevis del Gelnhausen, si inserisce nella schiera di coloro che scongiurarono la convocazione del concilio dal primo momento dell' apparire del suo fantasma. Si sa che i primi a chiedere il concilio o almeno a lanciarne l'idea del concilio nella ricerca di una soluzione non allo scisma, perchè ancora non attuato, ma alla questione della validità o meno dell'elezione del Prignano, furono i cardinali italiani nell'agosto del 1378, quattro mesi dopo l'elezione di Urbano VI. Motivi di ordine giuridico fecero saltare questa proposta che in seguito si fece strada sempre più energicamente. Ameilh come altri ${ }^{65}$, doveva scongiurare il concilio perchè non solo avrebbe messo in questione la duplice elezione degli stessi cardinali nell'arco di cinque mesi, ma avrebbe fatto saltare il concetto ecclesiologico che ostinatamente il Flandrin e l'Ameilh difendevano strenuamente. Dagli scritti di quasi tutti i difensori di Clemente VII si evince un'idea ecclesiologica che dalla fondazione del collegio cardinalizio andava sempre più sviluppandosi e che i cardinali avevano fatto propria. La difesa estrema di questi autori verteva su due punti fondamentali: da una parte combattere il tentativo del concilio con l'arma giuridica evidenziando tutte le difficoltà se celebrato senza il papa e addirittura contro di lui; dall'altra parte affermare che la chiesa intera da loro rappresentata deve tener conto di ciò che essi dicono per poter sapere veramente quali dei due sia il vero papa. Questi due argomenti fondamentali si ritrovano precisamente nel trattato dell'Ameilh. All'Embrunensis interessa evidenziare l'impossibilità giuridica della celebrazione del concilio, fondando la maggior parte delle sue argomentazioni sul Decretum Gratiani e sulle decretali e affermare che compito della cristianità è quello di ascoltare le loro dichiarazioni fatte a nome di tutta la chiesa.

Abbiamo già notato che il trattato dell'Ameilh presenta una difficoltà pregiudiziale; mentre per il Gelnhausen il problema della presenza dei due papi era chiaramente vigente e dunque da risolvere, per l'Ameilh uno solo è il papa vero, Clemente VII, mentre il Prignano non è altro che un intruso. Non è possibile convocare un concilio per giudicare e condannare un papa legittimo. Così su questa idea fondamentale costruisce la prima parte del suo scritto attaccando l'idea conciliare come se coloro che invocano il concilio non tenessero conto della presenza del vero papa. Sempre da questa prima parte sembra risultare che il cardinale voglia affermare l'idea ecclesiologica prima-

65 Tra coloro che giudicarono irnpossibile il concilio adducendo problemi di carattere giuridico ebbe il primo posto, arche cronologico, PIETRO FLANDRIN, Tractatus de Schismate, in FR. BLIEMETZRIEDER, op. cit., 3-71; anche S. Vincenzo Ferreri ebbe una parte importante in questa disputa teologica col suo De modemo Ecclesiae Schismate ed, da A. SORBELLJ, Bologna 1906. 
ziale della chiesa. Ricordare che il papa in quanto capo della chiesa non può essere giudicato, nè tanto meno esser deposto da nessuno, mentre nel corso della storia si sono verificati casi contrari, in quanto il papa in nome della sua plenitudo potestatis ha potuto deporre e giudicare imperatori e re. Intaccare questa verità di fondo significa intaccare la funzione stessa del papato nella chiesa, garanzia della sua unità e dell'autorità ${ }^{66}$. Se dalla questione prettamente giuridica risulta l'idea primaziale della chiesa e del posto che in essa il papa occupa, tuttavia dalla parte finale dello scritto risulta un'altra idea di chiesa: Ameilh sembra vedere l'autorità non nel solo papa ma in questi e nel collegio cardinalizio con lui. La plenitudo potestatis non sta solo nelle mani del Pontefice ma risiede nella chiesa romana che $\grave{e}$ il collegio dei cardinali col papa. proprio quest'idea che il Gelnhausen ha voluto sfatare nel suo scritto e non tanto quella di rispondere a questioni giuridiche. Al preposto di Worms premeva distinguere chiaramente l'universalità della chiesa dalla chiesa romana. Il gioco è tutto in questo punto: quale rapporto esiste tra la chiesa intera e quella della sede apostolica? È questa l'intera chiesa o la rappresenta soltanto? Da chi è formata la chiesa romana? Questi interrogativi sussistono ai trattati dell'uno e dell'altro, e rispettivamente danno una differente risposta. L'Ameilh considera il collegio cardinalizio col papa la Chiesa Romana che rappresenta l'intera chiesa ${ }^{67}$, mentre Gelnhausen, come abbiamo visto, pone il concilio come organo rappresentativo dell'universitas fidelium. L'Ameilh riprende fondamentalmente tutta una visione del rapporto tra cardinali e papa che nei secoli XII e XIII è andata formulandosi soprattutto con l'apporto della discussione dei decretisti, che hanno formulato il pensiero primaziale del papato. Intimamente annesso a questo fu la questione della chiesa romana e quella del collegio cardinalizio. Ben presto la chiesa romana venne vista nel papa stesso con il collegio dei cardinali, con le sue relazioni con questi «senatori» all'interno della chiesa. La Chiesa formata da caput et membra aveva una chiara formulazione nel papa visto come caput e nel collegio cardinalizio come membra.

Il collegio cardinalizio nel corso dei secoli dalla loro prima formazione tipicamente senatoriale con compiti non solo liturgici, ma soprattutto di ordine governativo, deve la sua creazione al papa Leone IX (1048-1054), in quella

66 Come per il Gelnhausen così per l'Ameilh dobbiamo fare attenzione a non comprendere in moxlo distorto la sua rispettiva ecclesiologica. Il Gelnhausen non è stato un conciliarista in senso stretto, così nell'Ameilh non troviamo un assertore dell'idea ecclesiologica primaziale del primato papale, piuttosto un'idea oligarchica dell'autorità nella chiesa. Fermandosi soltanto alla sua esposizione nella prima parte del trattato ci si potrebbe convincere della retta comprensione ecclesiologica a differenza di quella conciliare, eppure la seconda parte presenta la vera idea sottostante al suo scritto: il primato nella chiesa non è dato solo a uno,ma al papa e ai cardinali con lui. Per questo cfr $\mathbf{O}$. PREROVSKÝ, Le idee oligarchiche nei difensori di Clemente VII, in Salesianum 22 (1960) 383-408.

67 Cfr le pagine 108-111.

Proyección intemacional de la iglesia hispana Hispania Sacra 51 (1999) 
fase di riforma della chiesa che prende il nome di «riforma gregoriana» da Gregorio VII che più tardi la porterà a termine con la «lotta» contro l'ingerenza imperiale. I cardinali ebbero un compito prettamente legato al progetto di riforma che i papi di quest'epoca portarono avanti. E sempre in questa fase è da vedere lo stesso decreto di Niccolò II (1059-1061) sull'elezione dei papi in cui i cardinali vescovi ebbero una funzione determinante che sarà ulteriormente rafforzata da Alessandro III (1159-1181) ${ }^{68}$. Un collegio che veniva a crearsi visibilmente intorno alla persona del Pontefice necessitava una chiarificazione dottrinale. Come doveva essere visto il collegio cardinalizio? Quale compito doveva assumere nella chiesa? Quale rapporto tra esso e il papa? Ciò che non avvenne nel secolo XI esploderà nei secoli XII e XIII. I cardinali saranno considerati sempre più strettamente collegati alla chiesa romana e compartecipi della plenitudo potestatis del papa. Così, l'Hostiense ${ }^{69}$ (Enrico di Susa) in base ad un'idea corporativa della chiesa vedrà il potere del papa partecipato $\mathrm{al}$ collegio cardinalizio ${ }^{70}$, in cui non si ha più un'idea «monarchica» dell'autorità ma «oligarchica». Un corpo partecipa di questa plenitudo potestatis del papa che deciderà sempre più de consilio fratum nostrorum, che non sarà una semplice consultazione ma una vera e propria decisione da prendere insieme all'intero collegio. Di quest'idea è la Glossa palatina ${ }^{71}$ scritta da un autore ignoto tra il XII-XIII secolo. L'idea oligarchica nella Glossa è portata all'estreme conseguenze. Il Papa ha giurisdizione in quanto presiede il collegio cardinalizio che rappresenta l'intera chiesa ${ }^{72}$. Solo in questo modo egli può avere autorità. Ma perché questo?

A sostegno di questa visione sussiste sempre il concetto dell'universalitá della chiesa. L'intera chiesa è superiore a quella particolare, per cui i cardinali

68 Sul rapporto cardinali-papa, cfr B.TIERNEY, op. cit., 68-84; G. ALBERIGO, Cardinalato e collegialità. Studi sull'ecclesiologia tra l'XI e il XIV secolo, Firenze 1969 da leggere con qualche riserva espressa da M. Fols, I compiti e le prerogative dei Cardinali Vescovi secondo Pier Damiani nel quadro della sua ecclesiologia primaziale, in Archivum Historiae Ponticiae 10 (1972) 25-105, in cui fa notare che il primo a parlare dei compiti specifici dei cardinali vescovi non fu Umberto di Silva Candida ma Pier Damiani. Inoltre, per una chiarezza scientifica del problema del rapporto tra papa e cardinali soprattutto nel secolo XI, cfz la critica dello stesso autore al libro di Alberigo, Papa e cardinali nel secolo XI. Una questione di metodo e una replica, in AHP 14 (1976) 383-416. Infine P. BARBAINI, art. cit., 253-266.

69 Cfr TIERNEY, 149-153 in cui riporta brani della sua Lectura in Quinque Decretalium Gregorianarum Libros (Parisiis 1512).

70 «dicti sunt Cardinales a cardine quasi cum Papa mundum regentes...unde et dictum est non iudicabis in singulari, sed iudicabitis in plurali, ut non solum Papa sed et Cardinales includerentur etiam in expressione plenitudinis potestatis» Lectura ad IV, XVII 3.

71 Cfr TIERNEY, 81-84.

72 «Quaero utrum solus Papa possit condere canones. Solutio: generalem legern de universali statu ecclesiae non potest sine Cardinalibus conderes, ibidem, 81 . 
sono superiori al papa, perchè orbis maior est urbe (l'orbe=chiesa intera, $u r$ $b e=p a p a$, insieme chiesa romana che rappresenta la chiesa intera). Le prerogative proprie dei cardinali sussistevano nel fatto che essi rappresentavano l'intera chiesa anche quando la sede petrina era vacante. Inoltre, il potere di eleggere il papa faceva sì che essi godessero di un'autorità intrinseca espressa nell'elezione del papa ${ }^{73}$ che necessariamente con loro doveva governare la chiesa.

Da una tale concezione non poteva risultare immune l'Ameith. La Chiesa romana è, per lui, il collegio dei cardinali col papa ${ }^{74}$. La motivazione classica è data dal rapporto cardinali-papa visto alla luce di quello tra canonici-vescovo nella diocesi. A partire da questa visione giuridica e teologica l'Ameilh contribuisce ulteriormente all'affermazione di questo principio che andando formandosi lungo un periodo di continua ricerca dottrinale trova ora un'ulteriore forza nelle sue affermazioni. Questi condensa in soli quattordici righe la sua ecclesiologia con una particolare abbondanza di citazioni delle decretali che servono a sostenere quanto vuole affermare.

Partendo dal termine ecclesia, l'autore del trattato opera un doppio passaggio: dal vescovo passa a parlare del capitolo della cattedrale col vescovo. Questi, per l'Ameilh, è nella Chiesa e la Chiesa in lui. Ora, è da capire cosa intende per «chiesa». Una formulazione del genere potrebbe essere interpretata ingenuamente, senza comprendere il vero significato di ciò che sta sotto. Quando l'Ameilh parla di chiesa cita due testi, uno dal Decretum: Scire ${ }^{75}$;

${ }^{73}$ Non ha influito poco quest'idea nei cardinali nei confronti di Urbano VI. L'elezione veniva vista quasi come una creazione di una soggetto che doveva pur sempre essere riconoscente per la sua scelta. Alcuni atteggiamenti dei cardinali nei confronti del Prignano e quelli del papa nei loro indicano chiaramente lo stato d'animo di queste persone influenzate da diverse concezioni.

74 Riportiamo il testo perchè particolarnente incisivo: «Plus dico quod papa et domini cardinales ita sunt in Romana ecclesia, quod eciam ipsi sunt eadem Romana ecclesia, que claves accepit et potestatem ligandi atque solvendi, XXIII. q. I. Omnibus, XCVII. di. Ecclesie ante fin[em]; siquidem de papa nullus dubitat, quoniam generaliter de quolibet episcopo verum est quod sit in ecclesia et ecclesia in ipso, VD. q. 1 Scire, de testa. Requisisti, ubi patet quod appellacione ecclesie intelligitur capitulum cum episcopo. Immo quodam modo appellacione ecclesie communius intelligitur collegium quam prelatus, e. ti. Relatum II., de privil. Per exempcionem, et hoc est indubitatum, quandocimque additur ecclesia expresso prelato, ut cum dicitur esse spirituale matrimonium inter ecclesiam et prelatum, de elec. Cum inter canonicos, et all[egato] c[apitulo] requisisti, et verb. signific. Cum clerici, et de Romano Pontefice et Romana ecclesia habes LXIII. di. Quia sancta...», p. 110-111.

I primi due testi citati si trovano nel Decretum rispettivamente C. XXIV, q.1, cap. 20: Omnibus; Dist. XCVII, q.1, cap. 1. Per il testo Omnibus, l'Ameilh cita la Causa XXII, mentre è XXIV.

75 C. VII, q.1, cap.7: Scire: «Scire debes episcopum in ecclesia esse, et ecclesiam in episcopo, et si qui cum episcopo non sit, in ecclesia non esse, et frustra sibi blandiri eos, qui pacern cum sacerdotibus. Dei non hentes obrepunt, et latentes apud quosdam communicare se credunt, quando ecclesia, qua catholica una est, scissa non sit, neque diuisas.

Proyección internacional de la iglesia hispana Hispania Sacra 51 (1999) 
l'altro dalle decretali: Requisisti ${ }^{76}$. Dalla seconda decretale risulta in modo particolarmente sufficiente che quando l'Ameilh parla di ecclesia indica la chiesa cattedrale simbolo dell'intera diocesi. Il vescovo diocesano entra in un rapporto stretto con quella chiesa da pensarlo come se fosse un matrimonio spirituale. La chiesa diocesana simboleggiata dalla chiesa cattedrale indica l'universitas fidelium con cui il vescovo ha stretto un rapporto sponsale. $\mathrm{Si}$ tratta fondamentalmente di un'immagine plastica per indicare una realtà fondamentale che si è instaurata tra il prelato e l'intera comunità diocesana. Ma è proprio qui che si sviluppa un altro interessante punto. La chiesa cattedrale viene identificata col collegio dei canonici. Chi simboleggia la cattedrale della diocesi è il corpo dei canonici che raffigurano l'intera diocesi. Per cui, il rapporto sponsale tra il vescovo e la diocesi è un rapporto fondamentalmente vincolante tra il capitolo cattedralizio e il prelato posto in esso ${ }^{77}$. Per questo l'Ameilh può dire che ubi pateat quod appellacione ecclesie communius inteligitur collegium quam prelatus ${ }^{78}$. Il collegio dei canonici rappresenta l'intera diocesi simboleggiata dalla chiesa cattedrale. Al centro dunque resta l'universitas fidelium come per il Gelnhausen, ma a differenza di questi l'Ameilh risolve la questione difformemente per cui la congregatio fidelium viene a identificarsi con il collegio stesso dei canonici e dunque per analogia a quello cardinalizio.

L'Embrunensis non ha fatto altro che trasferire l'idea che assimilava il concetto di chiesa a quello di «collegio» dei canonici col vescovo a quello dei cardinali col Papa. Conseguenza esplicita quasi logica è che la chiesa Romana, che è la chiesa universale, è il collegio dei cardinali col papa, per cui può fare il suo giuramento significativo, fidelis ero sancte Romane ecclesie dominoque meo Cle [menti] eiusque successoribus canonice intrantibus ${ }^{79}$. La santa Roma-

76 Decretali: Lib. III, tit.26,cap.15: Requisisti: «quoniam non est tanta communio inter episcopum, et inter cappellas, vel monasteria, seu alia pia loca, quanta est inter episcopum, et ecclesiam cathedralem: cui est spirituali coniugio copulatus, unde quae acquirit episcopus, non illis, sed isti tantum acquirit...». Il contesto di questa decretale è sulla questione di lasciti di beni alla chiesa particolare.

77 Decretali: Lib.I, tit.6, cap. 21: Cum inter canonicos: «..per mutuum consensum elegentium, et electi, quasi coniugale vinculum spiritualiter sit contractum...»

78 Decretali: Lib. III, tit. 26 , cap.12 Relatum:« ...Nomine autem ecclesiae non episcopus, vel successor clerici merientis, ubi est collegium clericorum, sed communis congregatio intelligitur...» Decretali Liber sextus: $L i b$. V, tit.7, cap.9 Per exemptionem: «Verum si canonici alicuius ecclesiae eximantur: ipsi soli canonici, non autem ecclesiae vel alii eius clerici sunt exempti».

79 Decretali: Lib. V, tit.40, cap.19 Cum clerici: «Cum clerici Placentini iuramentum prestant sub hac forma verborum: Ego talis ab hac hora in antea fidelis ero, et obediens Placentinae ecclesiae, et domino meo episcopo Placentino: Nos consulere voluistis, utrum intelligi debeat clerus totius dioecesis, an capitulum tantum ecclesiae cathedralis? Nos igitur taliter respondemus, quod per ecclesiae Placentinae vocabulum in forma iuramenti praescripti non debeat intelligi totius dioecesis clerus; et si capitulum intelligant ecclesiae cathedralis is tamen qui prestat hiusmodi iuramentum, episcopo tanquam capiti principaliter obligatur». Cosi pure, $L i b$. II, tit.24, cap.4: Ego $N$.: «Ego N. episcopus ab hac hora in antea fidelis ero sancto Petro, santaeque Romanae ecclesiae, dominoque meo Papae C. eiusque 
na chiesa, dunque, è il collegio dei cardinali ${ }^{80}$. Il potere trasmesso da Cristo alla chiesa romana è esercitato dai cardinali col papa. La giurisdizione su tutta la Chiesa è della chiesa romana e quindi di questo collegio che la rappresenta. Così, i cardinali hanno un potere proprio che neppure il papa può ridimensionare, una giuridiszione che è innata nella stessa corporazione della chiesa. La loro giurisdizione, secondo Ameilh, non è stata intaccata affatto dalla Ne Romani di Clemente $\mathrm{V}$, perchè non interessa l'esercizio della giurisdizione dei cardinali ma quello del papa che i cardinali possono pretendere durante la sede vacante.

L'ecclesiologia del cardinale Ameilh non è un'ecclesiologia primaziale, ma oligarchica. La Chiesa viene considerata la chiesa quasi come un corpo aristocratico nelle cui mani è posto il potere su tutta l'intera compagine ecclesiale. Il collegio cardinalizio è l'espressione perfetta della rappresentatività delle membra della chiesa, mentre il papa è il caput che non può essere disgiunto dal resto del corpo. Tuttavia, proprio questo collegio acquista un'importanza fondamentale perchè da solo detïene il potere di eleggere il successore di Pietro. In quanto opera dei cardinali, gli altri non possono entrare in questioni che riguardano solo loro e il loro operato. Da questo si spiega perchè spesso dallo scritto si evince l'accusa mossa dall'Embrunensis a coloro che vogliono giudicare l'operato dell'elezione di Urbano VI e di Clemente VII. Per l'Ameilh e per la sua concezione cio non può essere possibile in quanto problema che interessa l'intero collegio cardinalizio di cui i fedeli possono con serenità accettare quanto affermano. L'elezione del pontefice è di giurisdizione propria dei cardinali e nessun potere estraneo può entrare in esso ${ }^{81}$. Da quest'idea risulta come il fatto che l'elezione papale riservata ai soli cardinali da Niccolò II e da Alessandro III in poi abbia influito in questa visione oligarchica della plenitudo potestatis. L'Ameilh dipende in tutto da questo. L'atto dell'elezione del nuovo pontefice è vera e propria giurisdizione dei cardinali a cui nessuno è superiore. Per l'elezione essi godono piena potestà circa l'approvazione dell'elezione e della riserva della singolare autorità dell'eletto ${ }^{82}$. Come si vede, l'idea conciliare non è completamente lontana da quest'impostazione di pensiero. Sia il conciliarismo che la visione oligarchica della chiesa partono in

successoribus canonice intrantibus. Non ero neque in consilio, neque in facto, ut vitam perdat, aut membrum, vel capiakur mala captione».

$80{ }_{\ldots}$, negari enim non potest quin hic appelacione Romane ecclesie intelligi oporteat cardinales secundum Host [iensen] ibi.», p.111.

81 «Porro c[apitulum] Licet probat, guod cardinales, quando de eleccione contendunt, non habent superiorem, qui iudicare valeat inter eos discordantes, ubi sunt partes equales; sed ubi due partes eorum concordat, non oportet ulterius disputare», p. 109.

82 «Quarto, quoniam nedum circa eleccionem summi pontificis ipsi obtinet plenam potestatem, sed eciam habent circa ipsius eleccionis approbacionem et electi recepcionem singularem auctoritatem», ibidem.

Proyección internacional de la iglesia hispana Hispania Sacra 51 (1999) 
definitiva dall'idea ecclesiologica di chiesa come corporazione, come congregatio fidelium, ma a differenza del conciliarismo che vede il concilio come l'organo a cui viene demandato l'esercizio dell'autorità della chiesa, tale visione identifica l'universalità dei fedeli col collegio cardinalizio. È da dire che questo Ameilh l'aveva particolarmente compreso. La sua è stata un'esposizione di un pensiero ecclesiologico che risolvesse il continuo richiamo al concilio. Sostenendosi soprattutto con le decretali ha voluto difendere un diritto proprio che un concilio avrebbe in ogni caso fatto saltare.

È da domandarsi se la figura del papa in questo trattato occupa un posto più importante che in quello dello scritto del Gelnhausen. In definitiva, sembra che per il preposto di Worms il papa abbia un'importanza ecclesiologica più fondamentale che non per l'Ameilh. Ancora, in Gelnhausen troviamo un' ecclesiologia primaziale molto più sviluppata che nell'Embrunensis, in cui l'elemento giuridico è sopravvalutato a discapito della Sacra Scrittura citata molto meno rispetto alle più delle decretali e allo stesso Decretum.

\section{ALCUNE CONSIDERAZIONI CONCLUSIVE}

Lo scisma della chiesa in Occidente si è consumato tra la fine del XIV e $\mathrm{i}$ primi decenni del XV secolo, a cavallo di due secoli che furono pieni di fermento all'interno e fuori della Chiesa. La conclusione del secolo dell' «esilio avignonese» e dell'insorgere dell'idea di stato assolutistico e conseguentemente del tramonto del medioevo con il passaggio all'epoca del rinascimento non poteva avere altro esito che quello di una crisi all'interno della chiesa che produrrà effetti in ogni campo della stessa, sia a livello istituzionale che dottrinale. I trentanove anni della divisione furono l'immagine più emblematica della conseguenza di ciò che una politica ecclesiastica aveva creato. Tutte le premesse perchè questa nefanda catastrofe incombesse sulla chiesa erano ormai poste negli anni della permanenza del papa ad Avignone. Essa non soltanto contribuì a far diminuire l'importanza della figura universale del pontificato romano, visto oramai soggetto alla politica francese, ma creò tutte le premesse per quelo sviluppo successivo di centralizzazione e dunque di mondanizzazione della chiesa che nel secolo XV e la metà del secolo XVI vedrà fortemente svilupparsi. Da qui in poi il grido della maggior parte dei cristiani sarà quello di riforma che ingloberà caput et membra dove per caput sarà indicata sempre più la curia romana e la sua amministrazione. Lo scisma tra $i$ due secoli, segno di una situazione instabile all'interno della chiesa, troverà l'appoggio di principi e re che seppero trarre da esso vantaggi per la propria politica. L'universalità della chiesa e della funzione del papa ora viene a cadere, ciò che più conta è ormai l'assolutismo del proprio stato e del sovrano reg- 
nante. La chiesa incomincia ad avere un carattere più propriamente statale. II Papa viene visto più come un sovrano all'interno del proprio stato che può competere con gli altri solo per la sua funzione di capo e non per la sua presunzione universale. La fine della teocrazia universale medioevale sarà definitivamente debellata durante lo scisma. Da Costanza in poi, il papato dovrà impegnarsi in quell'opera di restaurazione della sua funzione contro nemici all'interno e all'esterno della chiesa che tenteranno di ridimensionarne le funzioni. Ma queste tendenze si presentano già prima e durante lo scisma. La tendenza di ridimensionare la giurisdizione del papato da Bonifacio VIII è andata sempre più affermandosi grazie all'apporto di autori e giuristi che hanno formulato un'ecclesiologia più o meno democratica, in cui l'antica visione «monarchica» del papato andava sempre più scemando per affermare quella del principio della democrazia nella chiesa. Una giurisdizione vera e propria per questi sarà solo quella del principe, mentre quella del Papa sarà controllata attraverso organi competenti come può esserlo il concilio. Concilio: parola magica del periodo previo e concomitante allo scisma. Esso doveva essere solo un organo per risolvere il problema della divisione e della scissione o doveva divenire argomento di una dottrina ecclesiologica nuova? Questo è il problema del periodo del 1378-1417. Se la riflessione del dibattito precedente allo scisma sulla funzione e sulla giurisdizione del papa nella chiesa aveva posto la questione del concilio in modo più o meno accentuato, lo scisma vedrà questa teoria affermarsi, ma senza diventare sovversiva. Essa lo diventerà solo dopo Costanza, a Basilea (1431-1449). Il conciliarismo sarà presente fattivamente nella chiesa con un nuovo scisma che il secolo XV vedrà consumarsi. L'Haec sancta di Costanza verrà ripresa a Basilea contro le iniziative di scioglimento $\mathrm{e}$ di trasferimento del concilio da parte di Eugenio IV. Se è vero che l'aver ripreso questo documento per una situazione nuova rispetto a quella per cui fu elaborato ed emanato significo la sua snaturazione e la sua incomprensione, è pur vero anche che esso poteva essere utilizzato contro il papato a favore del concilio per una intrinseca ambiguità contenutistica ${ }^{83}$. Questo è vero conciliarismo che provocò un ritardo nell'approfondimento dell'ecclesiologia fino al XX secolo. Solo dal Vaticano I in poi fino al Vaticano II si trattò di ecclesiologia. Neppure il concilio di Trento l'affrontò. Non troviamo nessun trattato ecclesiologico negli atti del tritendino. Eppure i riformatori avevano attaccato la Chiesa nella sua istituzione e struttura. Ma Trento non poteva farlo. I padri conciliari erano ancora divisi tra episcopalisti e curialisti.

83 Sull'interpretazione del decreto di Costanza è da leggere M. Fors, $l$ Concilio di Costanza nella storiografia recente, in La Civilita Cattolica 126 (1975),2, 11-27; ID., Il valore ecclesiologico del decreto "Haec Sancta del Concilio di Costanza, ibidem 138-152. Inoltre, H. JEDIN, Concilio episcopale o parlamento della Chiesa. Un contributo all'ecclesiologia dei concili di Costanza e Basilea, in Chiesa della fede e Chiesa della storia, Brescia 1'972,127-155.

Proyección internacional de la iglesia hispana Hispania Sacra 51 (1999) 
Ma il conciliarismo, tuttavia, produrrà ancora un altro male: il ritardo della riforma che la maggior parte dei membri della chiesa chiederà ma che non ci si troverà in accordo su quale organo poteva essere competente o almeno più idoneo per una progettazione. Il concilio era da escludere per non porre una tentazione contro il papato. Si pensi al conciliabolo di Pisa del 1511. Ma lo scisma aveva evidenziato un altro problema. Quel periodo vedeva venire sempre più in auge la collocazione dei cardinali all'interno della chiesa. Tutte le premesse dottrinali e giuridiche nel medioevo diverranno conseguenze distorte in questo periodo. Lo stesso scisma può essere attribuito in buona parte a quest'idea che accompagnava l'azione dei cardinali sia per la funzione del primato del papa che per la sua cooperazione all'esercizio della sua potestas. Costanza sarà l'espressione dell'universalità della chiesa espressa nella convocazione del concilio come suo organo rappresentativo, così come ritenevano coloro che con gli scritti lo auspicavano per risolvere la divisione. Il collegio cardinalizio subirà una sconfitta da una parte, d'altra però per tutto il secolo successivo e buona parte del secolo XVI si impegnerà nelle cose che tutto riguardano tranne quelle per cui la loro presenza nella chiesa è giustificata. Il grido «tiforma» sarà ancora più forte contro gli scandali della curia.

Il papato, quindi, dallo scisma esce fuori alquanto abbattuto. La sua opera sarà quella di riconquista della sua dignità all'interno e all'esterno della chiesa. Eppure soprattutto all'esterno di essa il papato del XV secolo si impegnerà in modo particolare. Ma anche questo è un risultato del secolo precedente. Se oramai il ministero del papato non sarà più visto nella sua dimensione universale, lo stesso papa sarà impegnato a salvaguardare il suo regno, il suo stato per avere quella libertà di azione così come gli altri principi l'avevano nei propri regni. Le lotte per le conquiste di indipendenze, le nomine dei cardinali tra i propri parenti saranno chiaramente il segnale della preoccupazione del papato di garantirsi libertà e sicurezza rispetto al potere dispotico dei principi e dei re e a quello dei cardinali di cui dovrà sempre diffidare dopo quanto è avvenuto con lo scisma.

Ma qual è il problema centrale? La giurisdizione. Basta scorrere i titoli dei trattati di ecclesiologia del secolo XIV e XV, ma come anche quelli del XVIII, per accorgersi che in ognuno di essi è presente inevitabilmente il termine potestas e dunque l'argomento sarà quello del de potestate. Tutta l'ecclesiologia si è concentrata su questa questione fondamentale sia per lo scontro con il potere statale sia per la questione del rapporto tra papa e cardinali. Il grande scontro è sulla giurisdiuzione e sulla potestà del romano pontefice. Perchè l'interesse centrale fu quello della giurisdizione? Una risposta a questa domanda può essere questa: coloro che scrissero di ecclesiologia in questo periodo furono fondamentalmente giuristi, esperti del campo giuridico. Papi e so- 
prattutto cardinali conoscono bene le decretali e il Decretum Gratiani. Tutta la loro trattazione verte su questa raccolta di legge o su queste decisioni concistoriali, in cui non sempre traspare una sana ecclesiologia. Il fatto che siano stati prettamente dei giuristi a scrivere di ecclesiologia fece perdere di vista il vero significato della chiesa e della funzione del papato. Quasi sempre si applicavano quelle leggi che erano andate formulandosi in un'epoca in cui l'universalità del papato e della chiesa era garantita dalla visione stessa dell'unità della società. Oramai il tempo tra il XIV e il XV secolo intaccherà questa visione inoltrando quella della nazione. Gli stessi autori che abbiamo presi in esame devono confrontarsi con questa mentalità. Anzi possiamo dire che l'Ameilh molto più del Gelnahusen è rappresentante di quest'idea giuridica che pervade qualsiasi argomentazione ecclesiologica. La sua è tutta una visione ecclesiale in cui maggior peso ha la funzione gubernandi della chiesa. Il pensare a partire dal Decretum e dalle decretali riempirà il suo trattato di citazioni legislative che vogliono solo salvaguardare la sua posizione e quella dei suoi colleghi in quel determinato periodo. Il Gelnhausen, invece, si vedrà costretto a citare decretali solo per rispondere, ma la sua costruzione ecclesiologica già rispecchia un tentativo nuovo di comprensione della chiesa che non esclude affatto il papato, anzi la sua richiesta del concilio è motivata proprio dal riportare a unità ciò che lo scisma aveva diviso. Il continuo ricorso alla Sacra Scrittura come fondamento della sua ecclesiologia è il tentativo più valido di comprendere l'ecclesiologia e molto più vicino alla nostra sensibilità. Il continuo ricorso alle fonti giuridiche, invece, sarà solo l'espressione della garanzia della propria posizione di privilegio. Le idee dell' Ameilh come quelle di tutti i suoi compagni di pensiero falliranno completamente con la convocazione di Costanza. Il pensiero ecclesiologico, e soprattutto conciliare del Gelnhausen saranno di valido aiuto per Costanza soprattutto per superare il momento della crisi conciliare, ma non per opporre al papato il potere superiore del concilio. Nel preposto di Worms non troviamo un conciliarista. II suo è stato solo un tentativo valido in ordine teologico e giuridico di risolvere il problema. Il suo interesse fu quello di invocare un tribunale per giudicare non il papa ma i due pretendenti al trono papale.

Proyección internacional de la iglesia hispana

Hispania Sacra 51 (1999) 